Passivity-Based Stabilization of a 1-DOF Electrostatic MEMS Model with a Parasitic Capacitance

by

Imiya Manjula Wickramasinghe, B.Sc., MPhil

A Thesis

in

Mechanical Engineering

Submitted to the Graduate Faculty

of Texas Tech University in

Partial Fulfillment of

the Requirements for the Degree of

Master of Science

in

Mechanical Engineering

Approved

Prof. Jordan M. Berg (Committee Chairman)

Dr. Tim Dallas

Dr. Seon Han

Fred Hartmeister, J.D., Ed.D.

Dean of the Graduate School

May, 2008 
(c)2008, Imiya Manjula Wickramasinghe 


\section{ACKNOWLEDGEMENTS}

First and foremost, I would like to express my deepest gratitude to my adviser Professor Jordan Berg for his support during my work. With his enthusiasm, his inspiration, and his great efforts to make things the best possible, he helped to make the research fun for me. Throughout my research period, he provided guidance, sound advice, good teaching, good company, and lots of good ideas. I would have never been here without him.

I am indebted to Dr. Sanjeeva Maithripala for paving the way for me to my dreams. The foundation he had laid already on the current topic of study has been a solid guidance for me to work on this phase of research.

Balasaheb Kawade, who I have never met personally, was very generous to provide me with the initial computer codes for ANSYS simulations and since then he has always been available for me for the consultation needed. I wish to thank Bala for being so helpful to me.

I am very grateful to Dr Tim Dallas and Dr Seon Han for being available as the members of my thesis committee and also for supporting my graduate studies.

I wish to express my gratitude to Professor Kirthi Walgama, who taught me many things that still help me in my life and career. I wish to thank my dear friend Dr. Asanka Maithripala for extending help whenever needed and for his friendship.

I wish to thank my friends Asiri Gunathilaka, Hemal Dahanayaka, Nadeeka De Silva, Parakrama Ekanayake, Rochana Meegaskumbura, Nenad Stojanovic and Ganapathy Sivakumar for supporting me in many ways during my time in Texas Tech and Lubbock Sri Lankan community for their friendship.

Lastly, and most importantly, I wish to thank my wife, Sandamali. She has been supporting and taking care for me. 


\section{TABLE OF CONTENTS}

ACKNOWLEDGEMENTS ....................... ii

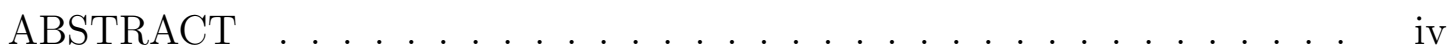

LIST OF TABLES . . . . . . . . . . . . . . . . . . V

LIST OF FIGURES . . . . . . . . . . . . . . . . . . v vi

1. INTRODUCTION . . . . . . . . . . . . . . . . . . 1

2. EQUILIBRIUM STRUCTURE OF A 1-DOF ELECTROSTATIC MEMS

MODEL WITH PARASITICS . . . . . . . . . . . . . . . 4

2.1 Introduction . . . . . . . . . . . . . . . . 4

2.2 A 1-DOF MEMS Model with Parasitic Capacitance . . . . . . 6

2.3 Voltage and Charge Pull-In . . . . . . . . . . . . . . . . . . . . 10

2.4 Passivity-Based Control Incorporating Parasitics . . . . . . . . 12

2.5 Simulation Results . . . . . . . . . . . . . . 15

2.5.1 Stability and Domain of Attraction for Case II . . . . . . . 15

2.5.2 Controller Validation using Matlab Simulation . . . . . . . 17

2.6 Conclusion . . . . . . . . . . . . . . . . 17

3. PASSIVITY-BASED STABILIZATION OF A 1-DOF ELECTROSTATIC MEMS MODEL WITH A PARASITIC CAPACITANCE . . . . . . 21

3.1 Introduction . . . . . . . . . . . . . . . . . . . 21

3.2 A 1-DOF MEMS Model with Parasitic Capacitance . . . . . . 23

3.3 Passivity-Based Control Design and Analysis Incorporating Parasitics . . . . . . . . . . . . . . . . 26

3.4 Simulation Results . . . . . . . . . . . . . . . . 32

3.4.1 Stability and Domain of Attraction for Case II . . . . . . . 32

3.4.2 Simulation of Cases I and II with Modeling Errors . . . . . 36

3.5 Conclusion ...................... 38

4. CONCLUSIONS . . . . . . . . . . . . . . . . . . 41

BIBLIOGRAPHY . . . . . . . . . . . . . . . . . . . . 42

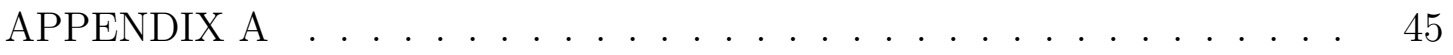

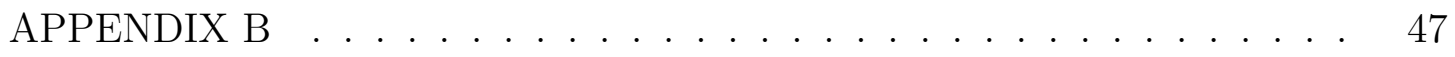

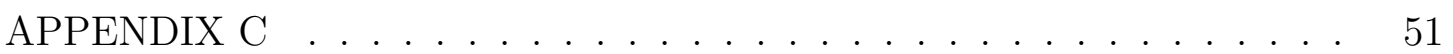




\begin{abstract}
This thesis addresses the problem of stabilizing 1-DOF piston mode electrostatic actuator in the presence of parasitic capacitance due to conductive substrate. The current study makes use of passivity-based control technique to formulate controllers. The static and dynamic controller schemes based on total charge can result in a unique equilibrium, however, their region of attraction may be small and the equilibrium may lose stability through Hopf bifurcation for certain configurations. A new charge quantity $\tilde{Q}_{c c}$ is introduced and used to derive static and dynamic feedback controllers in order to resolve issues encountered in the controllers based on total charge. The controllers based on $\tilde{Q}_{c c}$ are proved to be capable of globally asymptotically stabilizing the unique feasible equilibrium point for the configurations where the movable electrode is screened from the parasitic electrode by the control electrode. When the movable electrode and the parasitic electrode are directly coupled to have a mutual capacitance, numerical simulations show that the region of attraction of the closed-loop equilibrium is large. The effects of the infinite parallel plate approximation inherent in the formulation on the controller performance are investigated.
\end{abstract}




\section{LIST OF TABLES}

2.1 Expressions appearing in (2.1)-(2.5) for the two electrode geometry cases. . . . . . . . . . . . . . . . . . . 9

2.2 Equilibrium conditions with charge $Q_{c}$ as output. . . . . . . . . 11

2.3 Equilibrium conditions with total charge $Q_{e}$ as output. . . . . . . . 13

3.1 Function values for the two distinct electrode geometries. $\rho \equiv A_{0} / A_{c}$. $d$ is the zero-voltage gap. $\tilde{C}_{c c}$ is the capacitance between the control electrode and the grounded movable electrode. The term $C^{c p}$ arises from the inverse capacitance matrix. It is included here for completeness even though it is the same for both cases. . . . . . . . . . . . . 27

3.2 Zero dynamics equilibrium conditions with output $y=\tilde{Q}_{c c}-\overline{\tilde{Q}}_{c c} . \quad . \quad 29$ 


\section{LIST OF FIGURES}

2.1 1-DOF model of a electrostatic microactuator. The top plate of the MEMS is free to move and the two bottom plates are held fixed. The middle plate is the control electrode and the bottom plate is the parasitic electrode. This particular configuration is an example of Case II. The area of the bottom plate may be reduced to that of the top plate without affecting the simple capacitance models used for control design. The parasitic bias voltage $u_{p}$ is assumed to be zero in the controller design and analysis. . . . . . . . . . . . . . . . . .

2.2 Bifurcation diagram for the zero dynamics of a 1-DOF MEMS model without parasitics. Solid lines denote stable equilibria, dashed lines denote unstable equilibria. a) Voltage control. b) Charge control. . . 10

2.3 Equilibrium curves for the zero dynamics of a 1-DOF MEMS model under charge control with parasitics as parameter $\rho d / \delta$ varies. . . .

2.4 A complex conjugate root crosses into the right half-plane, signaling a

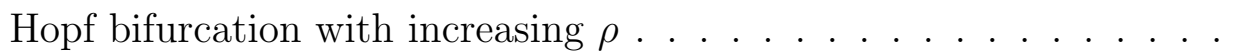

2.5 The displacement of the movable electrode corresponding to point 2 of figure $2.6 \ldots \ldots \ldots \ldots$. . . . . . . . . . . . . . . . 16

2.6 A complex conjugate root crosses into the right half-plane, signaling a

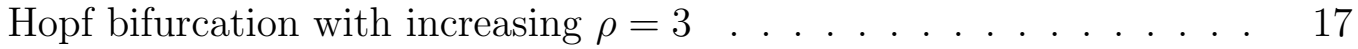

2.7 The displacement of the movable electrode corresponding to point 1 of figure $2.6 \ldots \ldots \ldots$. . . . . . . . . . . . . . . . . . . . . . . 18

2.8 Charge Pull-In with $Q_{c}$ control for a device of the type of case II . . .

2.9 Performance of the movable electrode for the controller (2.18) for a device of the type of case II . . . . . . . . . . . . . . . . . . . . . . 20 
3.1 1-DOF model of a electrostatic microactuator. The top plate of the MEMS is free to move and the two bottom plates are held fixed. The middle plate is the control electrode and the bottom plate is the parasitic electrode. This particular configuration is an example of Case II, as defined in the text. The area of the bottom plate may be reduced to that of the top plate without affecting the simple capacitance models used for control design. The parasitic bias voltage $u_{p}$ is assumed to be zero in the controller design and analysis. . . . . . . . . . . .

3.2 Bifurcation diagrams for the equilibrium curves $-x^{*}\left\{\mu_{I, I I}\left(x^{*}+1\right)+\right.$ $1\}^{2}=\gamma_{I, I I}$ of the zero dynamics of a 1-DOF MEMS model under charge control in the presence of parasitics, as parameter $\mu_{I, I I}$ varies. Curves correspond to $\mu_{I, I I}=0,0.5,1,1.5$, and 2. For $\mu_{I, I I} \geq 0.5$, charge pull-in occurs at the points marked by an ' $x$.' Stable branches are solid lines, unstable branches are dashed. For Case I, $\mu_{I}=d / \rho \delta$ and $\gamma_{I}=\bar{Q}_{c}{ }^{2} / 2 \epsilon A_{0} m \omega^{2} d$. For Case II, $\mu_{I I}=d / \delta$ and $\gamma_{I I}=\rho \bar{Q}_{c}{ }^{2} / 2 \epsilon A_{0} m \omega^{2} d .28$

3.3 Simulation results for Case II with static output feedback for three values of $\bar{x}$, with $\delta=0.5$ and $\rho=3 \ldots \ldots \ldots$

3.4 Simulation results for Case II with dynamic output feedback for four values of $\bar{x}$, with $\delta=0.5$ and $\rho=3 \ldots \ldots \ldots$

3.5 Comparison of parallel plate capacitance approximations to capacitances computed using $A N S Y S$. The movable electrode is $100 \mu \mathrm{m}$ by $200 \mu \mathrm{m}$, the control electrode is $57.7 \mu \mathrm{m}$ by $115.5 \mu \mathrm{m}$, and the parasitic electrode is $135 \mu \mathrm{m}$ by $270 \mu \mathrm{m}$, corresponding to $\rho=3$. The parasitic plate spacing $\delta$ is $0.5 \ldots \ldots \ldots \ldots \ldots \ldots$

3.6 Simulation of dynamic output feedback control of Case II, comparing use of the parallel plate approximation to use of capacitances and forces computed using ANSYS. The movable electrode is $100 \mu \mathrm{m}$ by $200 \mu \mathrm{m}$, the control electrode is $57.7 \mu \mathrm{m}$ by $115.5 \mu \mathrm{m}$, and the parasitic electrode is $135 \mu \mathrm{m}$ by $270 \mu \mathrm{m}$, corresponding to $\rho=3$. . . . . . . . 
3.7 Simulation of static output feedback control of Case I, comparing the parallel plate approximation to capacitances computed using ANSYS. The movable electrode is $100 \mu \mathrm{m}$ by $200 \mu \mathrm{m}$, the control electrode is $112 \mu \mathrm{m}$ by $223 \mu \mathrm{m}$, and the parasitic electrode is $162.4 \mu \mathrm{m}$ by 323.4 $\mu \mathrm{m}$, corresponding to $\rho=0.8 \ldots \ldots \ldots \ldots$

3.8 Simulation of dynamic output feedback control of Case I, comparing the parallel plate approximation to capacitances computed using $A N$ $S Y S$. The movable electrode is $100 \mu \mathrm{m}$ by $200 \mu \mathrm{m}$, the control electrode is $112 \mu \mathrm{m}$ by $223 \mu \mathrm{m}$, and the parasitic electrode is $162.4 \mu \mathrm{m}$ by 323.4

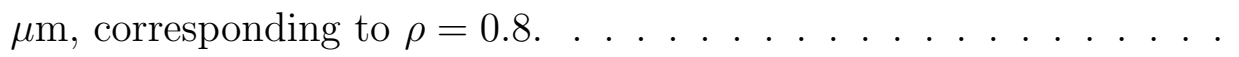

3.9 Simulation of static output feedback control of Case II, comparing the parallel plate approximation to capacitances computed using ANSYS. The movable electrode is $100 \mu \mathrm{m}$ by $200 \mu \mathrm{m}$, and the control and parasitic electrodes are $57.7 \mu \mathrm{m}$ by $115.5 \mu \mathrm{m}$ and $135 \mu \mathrm{m}$ by $270 \mu \mathrm{m}$ respectively, corresponding to $\rho=3$. . . . . . . . . . . . . 40

4.1 Configurations for Case I and Case II . . . . . . . . . . . . 45 
Texas Tech University, Imiya Manjula Wickramasinghe, May 2008

\section{CHAPTER 1 \\ INTRODUCTION}

Electrostatic actuation is often chosen for MEMS devices due to number of reasons such as compactness. In applications where the position of an electrode in a capacitive gap is to be controlled by electrostatic actuation the fundamental nature of pull-in has to be dealt with. Simple voltage control on such a device would provide stability only over one third of the gap. This limitation can be overcome by charge control [15]. However, presence of parasitic capacitances may destabilize the charge controller.

The structure of this thesis is as follows: the text of a refereed conference publication arising from the work [10] is presented in Chapter 2 (entitled ASME IMECE Paper, and referred to as Paper 1), and that of an archival, refereed, journal paper [24] is presented in Chapter 3 (entitled IEEE TCST Paper, and referred to as Paper 2). This introduction places the papers in the context of a larger research effort at Texas Tech, and updates the literature surveys to reflect recent work.

The research reported in this thesis is a continuation of previous work by Maithripala et al, as presented in [6], [7], [8] and [9]. Contributions by the coauthors are gratefully acknowledged. The framework of the approach was largely established by the previous work including the proposal for total charge based control to eliminate charge pull-in. The work of this thesis addressed two problems in the previous work on control in the presence of parasitics. First, inconsistencies in the treatment for different electrode geometries was rectified during the present study. Here the dynamic system is parametrically described independent of the size of the parasitic electrode area and then the parameters are specified for two distinct configurations of parasitic geometries. Next the investigation on the total charge approach led us to identify and rectify major limitations. That work is presented in Chapter 2. The controller based on total charge fails when the parasitics are sufficiently large. In that case, the resultant equilibrilia are unique but become unstable through subcritical Hopf bifurcation. We also noticed that, for larger parasitic case referred to as Case II in the text, the measurements of the voltage or charge on the parasitic structure are required to implement the total charge control. 
Texas Tech University, Imiya Manjula Wickramasinghe, May 2008

Thus the controller implementation becomes impossible in practice.

The process of investigation to solve the above mentioned problem of implementing total charge control led us to a new charge quantity, $\tilde{Q}_{c c}$. The $\tilde{Q}_{c c}$ based controller, which can be implemented using feasible measurements, is capable of stabilizing almost any geometric configuration of practical interest. This new formulation is presented in Chapter 3.

The literature surveys in Paper 1 and Paper 2 were done before 2006 and therefore it is necessary to look at recent research in the related fields. It was apparent that, among published research works the most relevant work during the past two years is found in [18] and it deals with the stabilization of 1 DOF model using input-to-state and robust backstepping techniques in the presence of parasitics. Also, [18] has further elaborated the nature of the parasitics treated in the formulation by considering series and parallel capacitive parasitics. The series capacitive parasitics have been used to explicitly model fringing field effects. Further, [18] models parametric uncertainties on physical parameters of the actuators and uses two alternative robust control schemes to deal with uncertainties. In contrast, parametric uncertainties are not addressed in the present study and the effects of fringing fields are investigated only through numerical simulations. On the other hand, the passivity-based controllers used in the present study enjoy the advantage of simplicity of control law over the robust controller schemes proposed in [18].

The other literatures that relate to pull-in phenomena during this period do not explicitly address the treatment of parasitics. [21] discusses theoretical MEMS model described by a reaction-diffusion equation and go on to estimate touchdown time. [22] investigates failures of capacitive MEMS switches due to parasitic charges and fundamental forces acting on such devices under dielectric charging. [23] presents an investigation on how pull-in voltage may be reduced by using special input signal patterns. That study highlights the effects of dynamics of the mechanical system on the pull-in voltage in contrast to quasi-static analysis, in which dynamics are not included. Further it suggests making use of stored mechanical energy in order to lower the voltage barrier for pull-in. These publications lack in relevance to the present study because of two reasons; first for not having modeled the parasitics explicitly and secondly owing to concentration on 
applications that make use of pull-in phenomena. 
Texas Tech University, Imiya Manjula Wickramasinghe, May 2008

\section{CHAPTER 2 \\ EQUILIBRIUM STRUCTURE OF A 1-DOF ELECTROSTATIC MEMS MODEL WITH PARASITICS}

\subsection{Introduction}

Electrostatic actuation of microelectromechanical systems (MEMS) makes use of the attractive coulomb forces that develop between capacitively-coupled conductors differing in voltage. Electrostatic actuation is nonlinear, making open-loop control over a large operating range difficult. Furthermore, the nonlinearity gives rise to a saddle-node bifurcation known as voltage pull-in that necessitates operational limitations. Eliminating this effect would allow for enhanced functionality in a number of applications by increasing the operational range of the movable electrode, reducing the need for motion limiters and anti-stiction measures, and preventing disturbances from causing the movable electrode to depart from its stable operating region.

A number of controls approaches have been presented in the literature to address pull-in. In the context of the current paper the most relevant are those that -implicitly or explicitly - make use of the substantial improvements in stability associated with control of electrode charge versus control of electrode voltage. Some notable examples include $[11,15,17]$. However, an additional challenge is to implement these controllers in the presence of dynamics arising from resistive and capacitive coupling both between device components, and between these components and the surroundings. These interactions are commonly referred to as parasitics. It is known that the effects of parasitic capacitance can cause loss of stability of charge-controlled electrostatic MEMS through a saddle-node bifurcation known as charge pull-in $[4,5,13,15]$. Explicit compensation of parasitic effects is only now beginning to attract attention from the controls community, for example [18].

In $[6,7,8,9]$ we present a series of results on passivity-based global and semi-global stabilization of electrostatically actuated MEMS. The one-degree-of-freedom case is considered in [6]. The system input and output are the control voltage applied to the fixed electrode and the charge on the movable electrode, respectively. Two controllers are derived that eliminate the pull-in 
Texas Tech University, Imiya Manjula Wickramasinghe, May 2008

bifurcation and stabilize any point in the capacitive gap. These are the only points for which a feasible equilibrium exists. One controller, based on the energy-shaping version of passivity [14], results in a charge feedback controller. The other, based on feedback passivation techniques [3], requires an additional velocity feedback term. Unlike the energy-shaping controller, the feedback-passivation controller may be used to inject damping into the mechanical subsystem, improving transient performance. The generalized model and controller presented in [7] extends the 1-DOF results to a broad class of electrostatically forced mechanical systems, including a variety of interesting MEMS devices. That extension requires measurement of the voltage and charge associated with all electrodes, including parasitic surfaces. Typically this is not feasible. When these measurements are not available the controllers of $[6,7]$ may fail to stabilize equilibrium points low in the gap, with loss of stability due to charge pull-in. The main object of the present note is to examine whether the global 1-DOF result of [6] may be recovered in the presence of parasitics without requiring charge or voltage measurements on the parasitic electrodes. The analysis presented here considers only stabilization and not improved transient behavior. The energy-shaping controller and the feedback-passivation controller are equivalent with respect to the location and stability of closed-loop equilibrium points. However so far only the behavior of the energy-shaping controller has been thoroughly studied. The feedback-passivation controller may provide a larger domain of attraction, and may influence the loss of stability through dynamic mechanisms such as Hopf bifurcation. This question is a topic of current research, and further discussion is beyond the scope of the present note.

The model considered is the standard 1-DOF model used in [6] - a grounded movable electrode suspended by a spring and damper above a fixed control electrode - with an additional fixed parasitic electrode added below the control electrode (see Fig. 2.1). As in $[6,7,1]$, the voltage across the movable and fixed electrode is assumed to be measured, along with either the charge on the fixed electrode or the mutual capacitance between the movable and fixed electrodes. In the present note the parasitic electrode is connected to ground through a resistor. Therefore both the parasitic voltage and the charge can vary dynamically. At 
Texas Tech University, Imiya Manjula Wickramasinghe, May 2008

equilibrium however the parasitic voltage will be zero. This condition may be relaxed by including a voltage source in series [8], but such an extension is beyond the scope of the present note. Physically the parasitic surface might represent the wafer substrate upon which the MEMS device is fabricated.

It is shown that feedback control using the total charge on the movable and parasitic electrodes as system output can prevent charge pull-in, and provide global asymptotic stability of the desired equilibrium. If the control electrode is bigger than either the movable electrode or the parasitic electrode then, neglecting fringing, the parasitic electrode is completely screened from the movable electrode. In this case the total charge feedback law can be implemented using the specified measurements. Otherwise the equilibrium is only locally asymptotically stable, and in fact the equilibrium may itself lose stability through a Hopf bifurcation. Furthermore, the feedback law can not be implemented using the specified measurements and will have to be approximated. This approximation does not affect the location of the equilibrium, but it may further reduce the region of attraction. These effects become more pronounced as the parasitic surface becomes larger with respect to the control surface, or as the parasitic surface moves closer to the control surface.

This note is organized as follows: Section 2.2 presents a 1-DOF model of an electrostatic MEMS with a parasitic capacitance. In Section 2.3 we revisit the nominal energy-shaping controller derived in $[6,7]$ and show how parasitics may cause charge pull-in, which we interpret in terms of bifurcation of the system zero dynamics. Section 2.4 demonstrates the use of total charge feedback to eliminates charge pull-in and recover global asymptotic stability, and examines how measured quantities may be used to implement this feedback. In Section 2.5 the controller is demonstrated using Matlab simulations. Finally, Section 2.6 summarizes the results and discusses possible extensions.

A preliminary version of these results was presented in $[8,9]$.

\subsection{A 1-DOF MEMS Model with Parasitic Capacitance}

We consider a MEMS device modeled by three parallel plates. On top is the movable electrode, suspended by spring and damping elements and constrained to translate in the vertical direction, in the middle is a fixed plate that we refer to as 


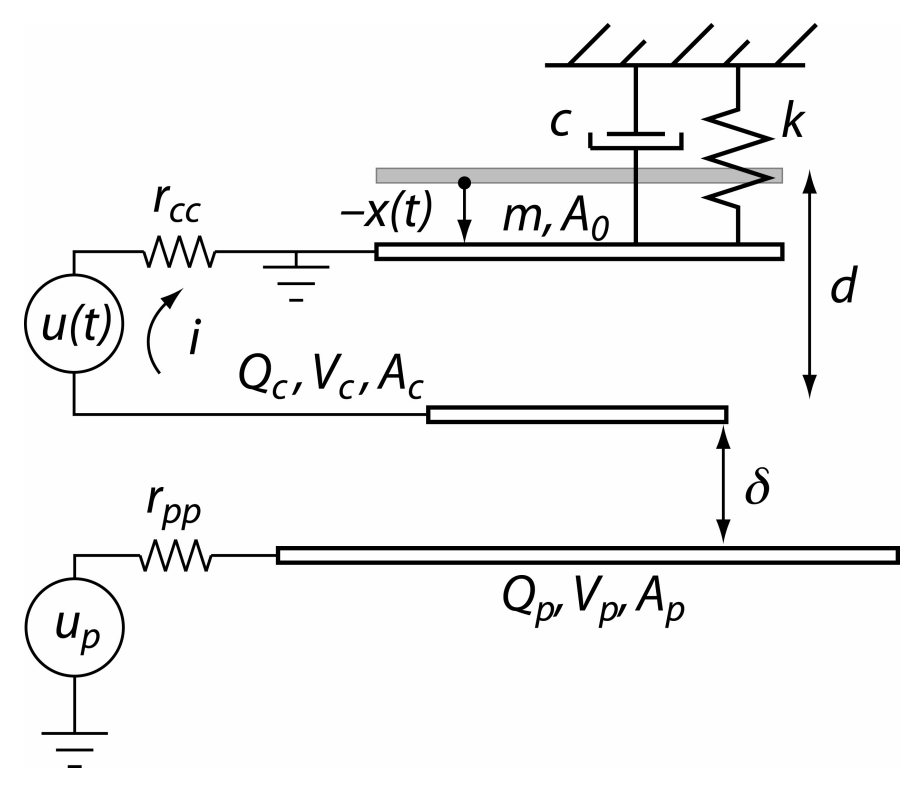

Figure 2.1. 1-DOF model of a electrostatic microactuator. The top plate of the MEMS is free to move and the two bottom plates are held fixed. The middle plate is the control electrode and the bottom plate is the parasitic electrode. This particular configuration is an example of Case II. The area of the bottom plate may be reduced to that of the top plate without affecting the simple capacitance models used for control design. The parasitic bias voltage $u_{p}$ is assumed to be zero in the controller design and analysis.

the drive or control electrode, and at bottom is a fixed parasitic electrode. The plates have area $A_{0}, A_{c}$, and $A_{p}$, respectively, and are assumed to be centered on a common axis. The zero voltage gap between the moving and drive electrode is $d$ and the distance between the parasitic and drive electrode is $\delta$. Resistive cross-coupling is neglected here, and the movable electrode is assumed to be grounded, that is, connected to ground through a zero voltage bias, with negligible series resistance. Figure 2.1 shows a schematic of the model. The configuration of the parasitic electrode is motivated by MEMS designs in which a parallel-plate device is surface micromachined on an insulating silicon dioxide or silicon nitride layer, which is in turn deposited on a (relatively conductive) silicon wafer. In such cases large parasitic capacitances may exist between the device and the underlying silicon, especially if the insulating layer is thin. In what follows, the parasitic bias 
voltage $u_{p}$ shown in Fig. 2.1 is assumed to be zero.

For the purpose of control design the capacitive coupling and electrostatic forces are derived using a simple infinite parallel plate model that neglects fringing. Thus the mutual capacitance between any two surfaces will be $C_{i j}=\epsilon A_{i j} / l_{i j}$, where $l_{i j}$ is the distance between the surfaces and $A_{i j}$ is the area of the overlap between the surfaces with no intervening conductor. If the parasitic effects are due to interactions with a conductive substrate, this model allows us to consider only that portion of the substrate directly beneath the device. $\epsilon$ is the permittivity of the material in the gap between the plates, sometimes written as a dielectric constant times the permittivity of free space. In what follows, $\epsilon$ is simply a constant.

Even using the simplified 1-DOF model of Fig. 2.1 several distinct electrode configurations are possible. The following observation facilitates the analysis: Under the simplified capacitance calculation, the largest electrode area may, without loss of generality, be reduced to that of the next largest, resulting in three possible situations, in which either the parasitic electrode is the smallest, the control electrode is the smallest, or the movable electrode is the smallest. The first and third are qualitatively very similar and the third may be easily treated by analogy to the first. Therefore, to avoid unnecessarily complex notation, we will consider only the first and second configurations, which we subsequently refer to as Case I and Case II. We define the parameter $\rho=A_{p} / A_{c}$. For Case I, $\rho \leq 1$ and for Case II $\rho \geq 1$. Note that in Case I the movable electrode is completely screened from the parasitic electrode by the control electrode. In Case II the movable electrode is directly affected by the parasitics.

The configuration space of the movable electrode is $G=\mathcal{R}$. Let $\left\{e_{1}\right\}$ be a coordinate frame for $\mathcal{R}$, with $e_{1}$ fixed at the center of mass of the moving electrode in zero voltage equilibrium and pointing away from the fixed control electrode, and with $x$ denoting the displacement of the movable electrode along $e_{1}$. Then application of the modeling procedure presented in $[7,8]$, gives the following 
equations of motion:

$$
\begin{aligned}
\dot{Q}_{c} & =-\frac{1}{r_{c c}} V_{c}+\frac{1}{r_{c c}} u_{c}, \\
& =-\frac{1}{r_{c c}} C^{c p}(x)\left(\alpha^{c c}(x) Q_{c}+Q_{p}\right)+\frac{1}{r_{c c}} u_{c}, \\
\dot{Q}_{p} & =-\frac{1}{r_{p p}} C^{c p}(x)\left(Q_{c}+\alpha^{p p}(x) Q_{p}\right), \\
\dot{x} & =v \\
\dot{v} & =-2 \zeta \omega v-\omega^{2} x-f^{e}\left(x, Q_{c}, Q_{p}\right),
\end{aligned}
$$

where $f^{e}\left(x, Q_{c}, Q_{p}\right)$ is of the form

$$
f^{e}\left(x, Q_{c}, Q_{p}\right)=\frac{1}{2 m \epsilon A_{0}}\left(f_{11}^{e}(x) Q_{c}^{2}+2 Q_{c} Q_{p}+Q_{p}^{2}\right)
$$

Charge and voltage are related by

$$
\left[\begin{array}{l}
V_{c} \\
V_{p}
\end{array}\right]=C^{c p}\left[\begin{array}{cc}
\alpha^{c c} & 1 \\
1 & \alpha^{p p}
\end{array}\right]\left[\begin{array}{l}
Q_{c} \\
Q_{p}
\end{array}\right]
$$

where $C^{c p}(x)=(x+d) / \epsilon A_{0}$, and the voltage $V_{c}$ on the drive electrode is assumed to be measured. The two electrode configuration cases differ only in $\alpha^{c c}, \alpha^{p p}$, and $f_{11}^{e}$. The situation is summarized in Table 2.1.

Table 2.1. Expressions appearing in (2.1)-(2.5) for the two electrode geometry cases.

\begin{tabular}{|c|c|c|}
\hline Case & I & II \\
\hline \hline Smallest Area & $A_{p}$ & $A_{c}$ \\
\hline Other Areas & $A_{c}=A_{0}$ & $A_{p}=A_{0}$ \\
\hline$f_{11}$ & 1 & $1+\frac{\delta^{2}(\rho-1)}{(x+d+\delta)^{2}}$ \\
\hline$\alpha^{c c}$ & 1 & $1+\frac{\delta(\rho-1)}{x+d+\delta}$ \\
\hline$\alpha^{p p}$ & $1+\frac{\delta}{\rho(x+d)}$ & $1+\frac{\delta}{x+d}$ \\
\hline
\end{tabular}




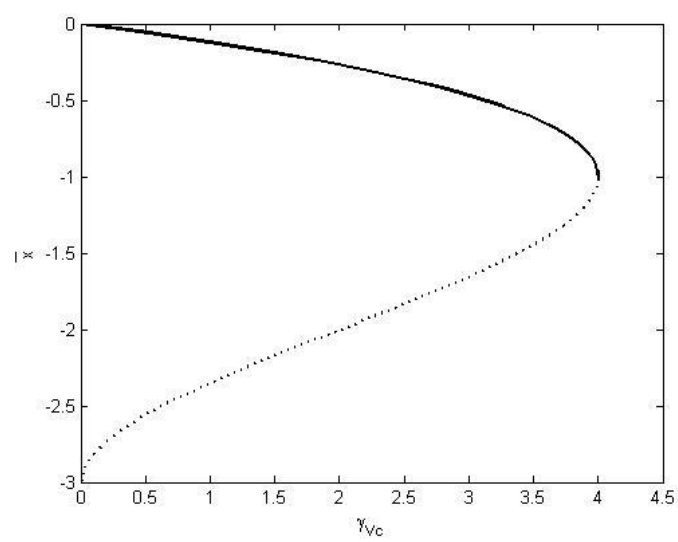

a)

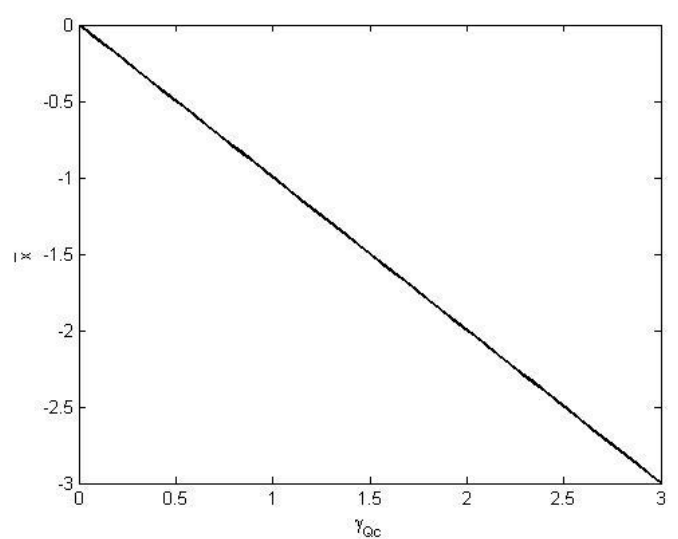

b)

Figure 2.2. Bifurcation diagram for the zero dynamics of a 1-DOF MEMS model without parasitics. Solid lines denote stable equilibria, dashed lines denote unstable equilibria. a) Voltage control. b) Charge control.

\subsection{Voltage and Charge Pull-In}

It is instructive to review the system without parasitics, and recall how the choice of charge as the controlled output eliminates the voltage pull-in bifurcation. In this case (2.3) is omitted, the $Q_{p}$ terms do not appear in (2.2), or (2.5), and $\alpha^{c c}(x)=f_{11}(x)=1$. The simplified capacitance models imply that, without loss of generality, $A_{c}$ and $A_{0}$ may be assumed equal to the smaller of the two, here arbitrarily denoted $A_{c}$. For the voltage control case the output is chosen as $y=V_{c}\left(x, Q_{c}\right)-\bar{V}_{c}=(d+x) Q_{c} / \epsilon A_{0}-\bar{V}_{c}$. Thus, at equilibrium, $y \equiv 0$ implies $\bar{Q}_{c}=\epsilon A_{0} \bar{V}_{c} /(d+\bar{x})$. The relative degree of the system is one, and the zero dynamics are the dynamics of the mechanical subsystem. Any equilibrium of the zero dynamics must satisfy $\bar{v}=0$ and

$$
-(\bar{x}+d)^{2} \bar{x}=\frac{\epsilon A_{0} \bar{V}_{c}^{2}}{2 m \omega^{2}} \equiv \gamma_{V_{c}}
$$

Figure 2.2(a) plots the roots of 2.7 that fall in the physical region $-d \leq \bar{x} \leq 0$ versus a bifurcation parameter, $\gamma_{V_{c}}$, which is simply the value of the right-hand side of 2.7 . A particular value of $\gamma$ corresponds to two, one, or no equilibrium points. When there are two equilibria the one closer to the origin is stable, the other is unstable, as 
shown in the figure. Voltage pull-in corresponds to the saddle-node bifurcation that occurs at $\bar{x}=-d / 3$, where there is a single equilibrium in the feasible region. For charge control the output is chosen as $y \equiv Q_{c}-\bar{Q}_{c}$. The zero dynamics are again the dynamics of the mechanical subsystem, now with equilibria satisfying $\bar{v}=0$ and

$$
-\bar{x}=\frac{\bar{Q}_{c}^{2}}{2 \epsilon A_{0} m \omega^{2}} \equiv \gamma_{Q_{c}} .
$$

Figure 2.2(b) plots the roots of (2.8) that fall within the feasible region versus the right-hand side, $\gamma_{Q_{c}}$. Now a particular value of $\gamma_{Q_{c}}$ corresponds either to a unique stable equilibrium point, or to no equilibrium point. The voltage pull-in bifurcation is eliminated. This observation, as well as being implicitly exploited in several previous studies such as $[11,15]$, was the basis for the 1 -DOF passivity controller in [6], and the motivation for the general method presented in [7].

Table 2.2. Equilibrium conditions with charge $Q_{c}$ as output.

\begin{tabular}{|c|c|c|}
\hline Case & I & II \\
\hline \hline $\bar{Q}_{p}$ & $-\frac{Q_{c} \rho(\bar{x}+d)}{\rho \bar{x}+\rho d+\delta}$ & $-\frac{Q_{c}(\bar{x}+d)}{\bar{x}+d+\delta}$ \\
\hline$f^{e}\left(\bar{x}, \bar{Q}_{c}, \bar{Q}_{p}\right)$ & $\frac{\delta^{2} Q_{c}^{2}}{2 m \epsilon A_{0}(\rho \bar{x}+\rho d+\delta)^{2}}$ & $\frac{\rho \delta^{2} Q_{c}^{2}}{2 m \epsilon A_{0}(\bar{x}+d+\delta)^{2}}$ \\
\hline
\end{tabular}

With the parasitic surface included in the model, it can be verified that for both Cases I and II voltage control gives zero dynamics equilibrium conditions identical to (2.7). Therefore voltage pull-in bifurcation occurs as before. Charge control, however, is fundamentally altered by the presence of the parasitics. The equation governing the equilibrium position of the electrode becomes for Case I

$$
-\left(\bar{x}+d+\frac{\delta}{\rho}\right)^{2} \bar{x}=\frac{\delta^{2} \bar{Q}_{c}^{2}}{2 \epsilon A_{0} m \omega^{2} \rho^{2}} .
$$

Comparing (2.9) to (2.7) we see that there will be a saddle-node bifurcation, referred to "charge pull-in." It occurs at at $\bar{x}=(d / 3)+(\delta / 3 \rho)$ which is greater than $d / 3$. If $\frac{\rho d}{\delta} \leq 1 / 2$ then charge pull-in does not occur in the physically feasible region. 
For Case II the equilibrium equations are

$$
-(\bar{x}+d+\delta)^{2} \bar{x}=\frac{\rho \delta^{2} \bar{Q}_{c}^{2}}{2 \epsilon A_{0} m \omega^{2}} .
$$

Once again charge pull-in occurs, this time at a critical displacement of $(d / 3)+(\delta / 3)$, again greater than $d / 3$. Therefore, as was first noted in [5], charge control confers a greater operating range than voltage control, even in the presence of parasitics. However, as $\delta$ becomes small or $\rho$ becomes large, any advantage becomes vanishingly slight. Figure 2.3 shows this behavior graphically as the parameter $\rho d / \delta$ varies.

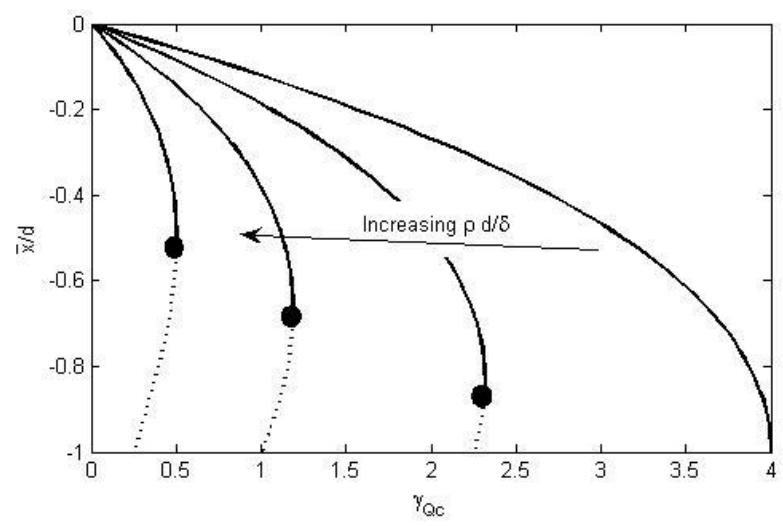

Figure 2.3. Equilibrium curves for the zero dynamics of a 1-DOF MEMS model under charge control with parasitics as parameter $\rho d / \delta$ varies.

From a passivity point of view, in the absence of parasitics the choice of charge as the output instead of voltage eliminates voltage pull-in. However parasitics induce the related phenomenon of charge pull-in. Then it is natural to ask whether there is another system output, for which charge pull-in does not occur. The next section demonstrates that there is.

\subsection{Passivity-Based Control Incorporating Parasitics}

In the absence of parasitics, $f^{e}\left(x, Q_{c}\right)$ in $(2.5)$ is $Q_{c}^{2} / 2 m \epsilon A_{0}$. With parasitics, for Case I, $f_{11}^{e}=1$, and so $f^{e}\left(x, Q_{c}, Q_{p}\right)=\left(Q_{c}+Q_{p}\right)^{2} / 2 m \epsilon A_{0}$. This suggests that 
Table 2.3. Equilibrium conditions with total charge $Q_{e}$ as output.

\begin{tabular}{|c|c|c|}
\hline Case & I & II \\
\hline \hline $\bar{Q}_{p}$ & $-\frac{\rho(\bar{x}+d)}{\delta} \bar{Q}_{e}$ & $-\frac{\bar{x}+d}{\delta} \bar{Q}_{e}$ \\
\hline$f^{e}\left(\bar{x}, \bar{Q}_{e}, \bar{Q}_{p}\right)$ & $\frac{Q_{e}^{2}}{2 m \epsilon A_{0}}$ & $\frac{\rho Q_{e}^{2}}{2 m \epsilon A_{0}}$ \\
\hline $\bar{x}$ & $-\frac{Q_{e}^{2}}{2 m \epsilon A_{0} \omega^{2}}$ & $-\frac{\rho Q_{e}^{2}}{2 m \epsilon A_{0} \omega^{2}}$ \\
\hline
\end{tabular}

choosing as system output the total charge $Q_{e} \equiv Q_{c}+Q_{p}$ will eliminate the charge pull-in bifurcation. This result is immediate for Case I. For Case II, the substitution $Q_{c}=Q_{e}-Q_{p}$ yields a less clear-cut expression:

$$
f^{e}\left(x, Q_{e}, Q_{p}\right)=\frac{Q_{e}^{2}}{2 m \epsilon A_{0}}+\frac{\delta^{2}(\rho-1)}{(x+d+\delta)^{2}} \frac{\left(Q_{e}-Q_{p}\right)^{2}}{2 m \epsilon A_{0}}
$$

However solving for $\bar{Q}_{p}$ in terms of $\bar{x}$ gives the same equilibrium equation as for Case I. These results are summarized in Table 2.3. In both cases the system with total charge as output has relative degree one and a unique equilibrium point. The elimination of charge pull-in suggests that any feasible equilibrium may be made globally asymptotically stable, but, as will be seen, this is not true. Three problems arise, all associated only with Case II. The first is that the arguments for global stability of the equilibrium no longer hold, and in fact simulations show that the region of attraction is finite. The second is that while the charge pull-in saddle-node bifurcation has been eliminated, the unique equilibrium may lose stability via a Hopf bifurcation. The third is that in this case $Q_{e}$ feedback is not implementable from the assumed measurements $x, V_{c}$, and $Q_{c}$. An approximation must be used, which further reduces the region of attraction.

We transform the system $(2.1)-(2.5)$ from $\left(Q_{c}, Q_{p}, x, v\right)$ to $\left(Q_{e}, Q_{p}, x, v\right)$ coordinates, and proceed as in [6] by applying the input-output linearizing control

$$
\begin{aligned}
u_{c}=r_{c c} & \left(\left(\frac{1}{r_{c c}}+\frac{\alpha^{p p}(x)}{r_{p p}}\right) V_{c}+\right. \\
& \left.\frac{C^{c p}(x)\left(1-\alpha^{c c}(x) \alpha^{p p}(x)\right)}{r_{p p}} Q_{c}+\nu\right)
\end{aligned}
$$


to obtain the following system:

$$
\begin{aligned}
\dot{Q}_{e} & =\nu \\
\dot{x} & =v \\
\dot{v} & =-2 \zeta \omega v-\omega^{2} x-f^{e}\left(x, Q^{e}, Q^{p}\right), \\
\dot{Q}_{p} & =-\frac{C^{c p}(x)}{r_{p p}}\left(\left(\alpha^{p p}(x)-1\right) Q_{p}+Q_{e}\right),
\end{aligned}
$$

where

$$
f^{e}\left(x, Q^{e}, Q^{p}\right)=\frac{1}{2 m \epsilon A_{0}}\left(Q_{e}^{2}+\left(f_{11}^{e}(x)-1\right)\left(Q_{e}-Q_{p}\right)^{2}\right)
$$

A stabilizing energy-shaping controller may be designed for Case I following [7],:

$$
\nu=-k\left(Q_{e}-\bar{Q}_{e}\right)
$$

where $k>0$. The choice of $Q_{e}$ as output ensures a unique equilibrium point for every $-d \leq \bar{x} \leq 0$. For Case I (2.13)-(2.15) reduce exactly to the equations without parasitics, with $Q_{e}$ replacing $Q_{c}$. Thus repeating the proof in [6] for this case shows that the controller (2.17) globally asymptotically stabilizes any desired feasible equilibrium. Implementation requires $Q_{e}$. For Case I we find from (2.6) the following expression for $Q_{e}$ in terms of $V_{c}$ and $x$ :

$$
Q_{e}=\frac{\epsilon A_{c}}{x+d} V_{c}
$$

Therefore, assuming availability of $x, V_{c}$, and $Q_{c}$, a static output feedback controller may be implemented for Case I as

$$
\nu=-k\left(\frac{\epsilon A_{c}}{x+d} V_{c}-\bar{Q}_{e}\right)
$$

Furthermore, as long as the various modeling assumptions are valid, the performance of Case I will be identical to the nominal case investigated in [6].

In Case II the expression for $Q_{e}$ also contains a term involving the parasitic voltage:

$$
Q_{e}=\frac{\epsilon A_{c}}{x+d} V_{c}+\frac{\epsilon A_{c}(\rho-1)}{x+d+\delta} V_{p}
$$


Thus it is infeasible to implement the $Q_{e}$ controller with available measurements for Case II. We propose to neglect the $V_{p}$ term in (2.20), and to approximate $Q_{e}$ in Case II by (2.18), that is, $\tilde{Q}_{e}=\epsilon A_{c} V_{c} /(x+d)$. So we obtain for Case II the same controller (2.19) as for Case I. Due to the assumption of zero parasitic bias voltage in the system model $(2.1)-(2.5), \bar{V}_{p}=0$. Therefore the use of (2.18) in steady state

gives the correct value of $\bar{Q}_{e}$, even for Case II. Then the results of Table 2.3 are still valid, and charge pull-in bifurcation will not occur. Next we note that for $\rho$ close to unity the $V_{p}$ term in (2.20) is small, and so it is reasonable to expect that in such situations the unique equilibrium will be stable and the region of attraction large.

In summary, we have shown in this section that the output feedback controller (2.19) eliminates charge pull-in for both Case I and Case II. For Case I we also have global asymptotic stability of the resulting unique equilibrium point. For Case II we have no stability guarantee, but for $\rho$ close to one we expect robust stability. Recall that under the simplified capacitance models used, the area of the parasitic surface can be taken in Case II to be equal to the area of the movable electrode. Therefore $\rho$ may be estimated as the ratio of the area of the movable electrode to the area of the drive electrode. In actual MEMS devices this is often close to one, and rarely greater than ten. The following section uses Matlab simulation to examine the stability of the equilibrium as $\rho$ increases.

\subsection{Simulation Results}

This section presents a set of simulation results. Section 2.5.1 shows the existence of a Hopf bifurcation in Case II with total charge feedback. We stress that this bifurcation occurs even when the exact expression for total charge is used. In section 2.5.2 we demonstrate using MATLAB simulations the stabilizing properties of the total charge feedback control developed in here.

\subsubsection{Stability and Domain of Attraction for Case II}

Figure 2.4 shows the root locus of the linearized zero dynamics of (2.13)-(2.15) as $\rho$ varies for fixed $\delta$. When $\rho$ changes from 1.80 to 1.85 a complex conjugate pair of roots crosses into the right half-plane, signaling a Hopf bifurcation. The locus of a single complex root is shown in Fig. 2.4. The behavior corresponding to the roots in 


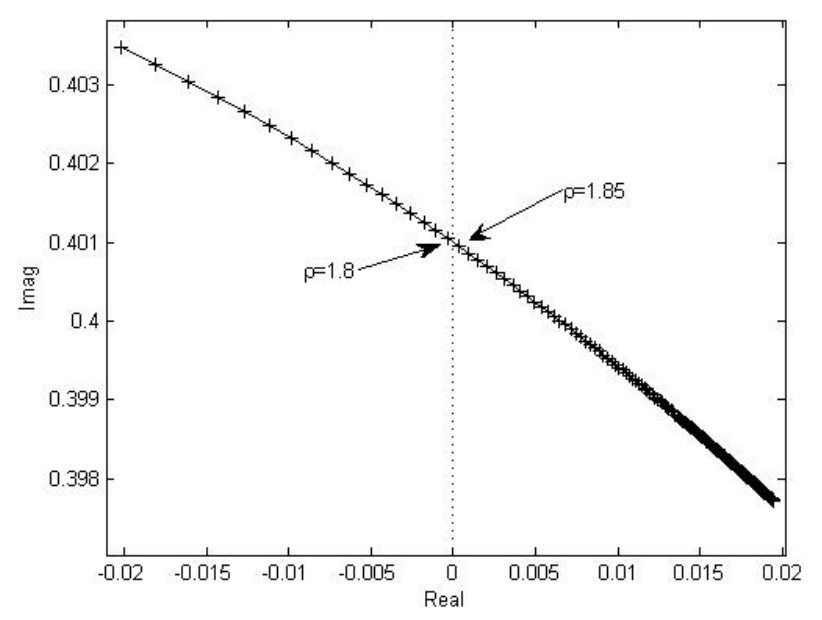

Figure 2.4. A complex conjugate root crosses into the right half-plane, signaling a Hopf bifurcation with increasing $\rho$

right half plane is oscillatory and grows to the limit of physical region as shown in Fig. 2.5. Figure 2.6 shows the regions of the $\rho$ - $\delta$ parameter space in which the Hopf

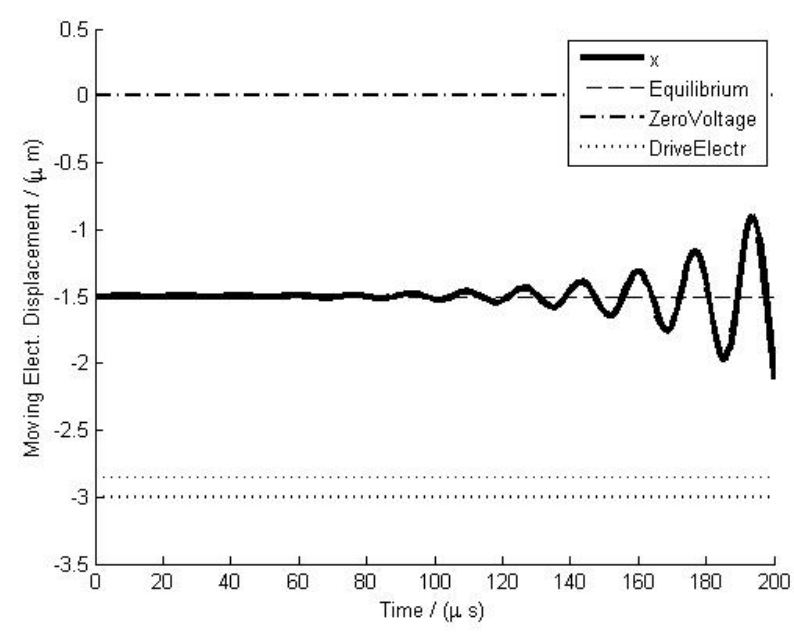

Figure 2.5. The displacement of the movable electrode corresponding to point 2 of figure 2.6

bifurcation occurs. This will depend on the natural damping of the system, here 


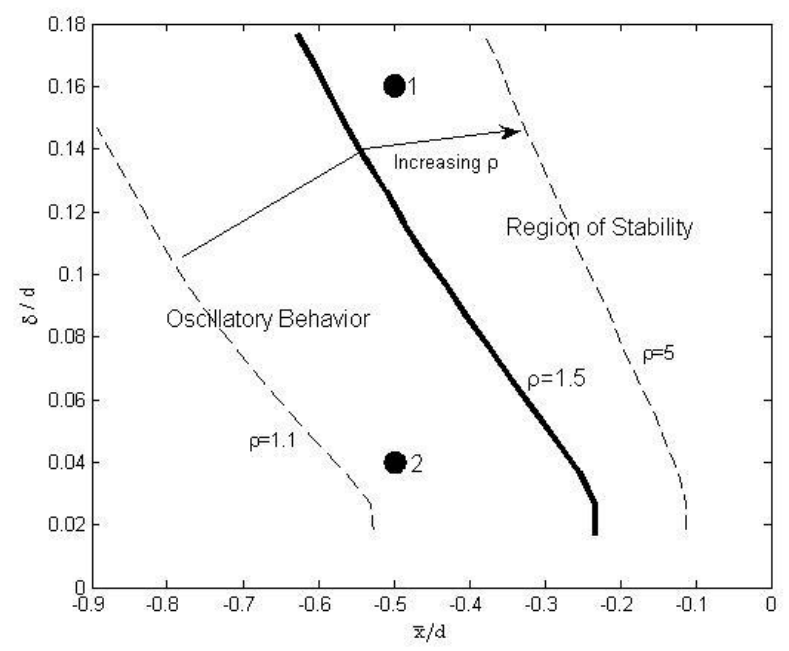

Figure 2.6. A complex conjugate root crosses into the right half-plane, signaling a Hopf bifurcation with increasing $\rho=3$

taken as 0.05. The line shown in Fig. 2.6 corresponds to the value of $\rho=1.5$ and the line is shifted to right with increasing $\rho$. The configurations corresponding to the points 1 and 2 of Fig. 2.6 yield the zero dynamics behaviors shown in Fig. 2.7 and Fig. 2.5.

\subsubsection{Controller Validation using Matlab Simulation}

Figure 2.8 shows how $Q_{c}$ control results in charge pull-in in the presence of parasitics. Figure 2.9 shows the controller (2.18) designed for Case I applied to a model with Case II geometry. The analysis of section 2.4 suggests that in steady state the controller designed for Case I gives the correct value of $Q_{e}$, even for Case II due to the assumption of zero parasitic bias voltage in the system model. The simulations support this conclusion, with performance excellent at $\rho=3$.

\subsection{Conclusion}

This paper presents a 1-DOF model including a capacitively coupled parasitic electrode, specialized from a general modeling and controls framework for electrostatically actuated MEMS. Previous work by the authors presented static and 


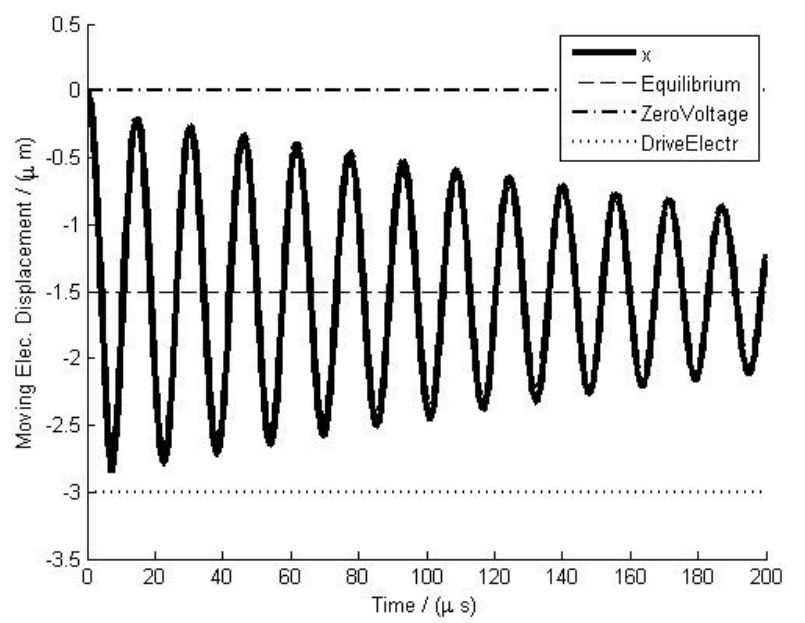

Figure 2.7. The displacement of the movable electrode corresponding to point 1 of figure 2.6

dynamic output feedback controllers that, in the absence of parasitics, globally asymptotically stabilize any equilibrium electrode configuration, thereby eliminating voltage pull-in and allowing the movable electrode an extended travel range. These controllers were based on the observation that the bifurcation that occurs in the zero dynamics when drive electrode voltage is chosen as the system output does not occur when drive electrode charge is chosen instead. However a sufficiently large parasitic capacitance will destroy this property, and a saddle-node bifurcation will again occur. This phenomenon, known in the literature as charge pull-in, limits the operational range of travel of the movable electrode, though less severely than voltage pull-in. For the 1-DOF model considered, we show that the use of total charge as the system output eliminates the charge pull-in bifurcation. Two qualitatively different electrode geometries must be considered. In the first, we recover the global asymptotic stability result obtained without parasitics. In the second, depending on the size of the parasitic capacitance, the region of attraction of the unique closed-loop equilibrium point is finite, and in fact it may lose stability through a Hopf bifurcation. Furthermore, in the second geometry it is not possible to implement total charge feedback with feasible measurements. An output feedback controller neglecting the unmeasurable quantities still eliminates the 


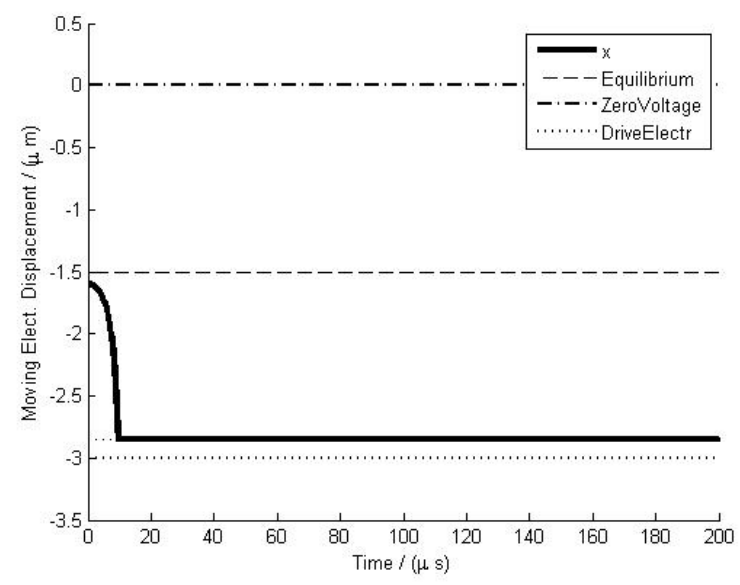

Figure 2.8. Charge Pull-In with $Q_{c}$ control for a device of the type of case II

pull-in, but further reduces the domain of attraction. 


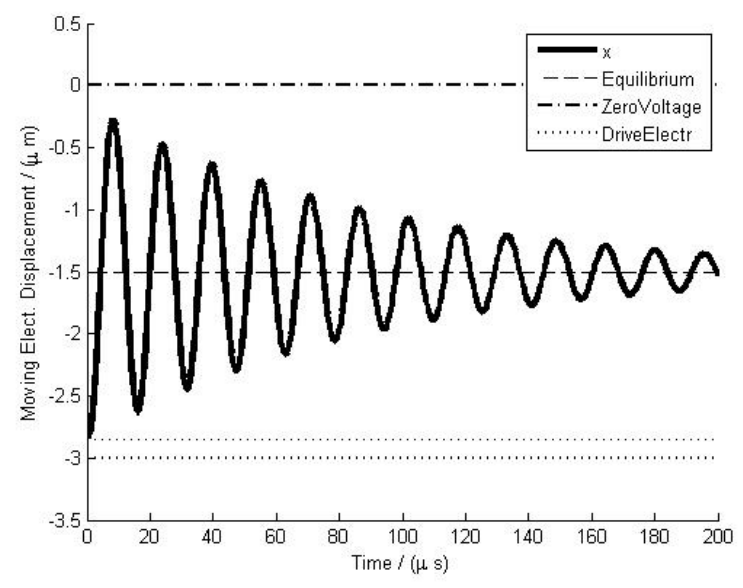

Figure 2.9. Performance of the movable electrode for the controller (2.18) for a device of the type of case II 
Texas Tech University, Imiya Manjula Wickramasinghe, May 2008

\title{
CHAPTER 3 \\ PASSIVITY-BASED STABILIZATION OF A 1-DOF ELECTROSTATIC MEMS MODEL WITH A PARASITIC CAPACITANCE
}

\begin{abstract}
3.1 Introduction
Electrostatic actuation of microelectromechanical systems (MEMS) makes use of the attractive coulomb forces that develop between capacitively-coupled conductors differing in voltage. Electrostatic actuation is nonlinear, making open-loop control over a large operating range difficult. Furthermore, the nonlinearity gives rise to a saddle-node bifurcation known as voltage pull-in that necessitates operational limitations. Eliminating this effect would allow for enhanced functionality in a number of applications by increasing the operational range of the movable electrode, reducing the need for motion limiters and anti-stiction measures, and preventing disturbances from causing the movable electrode to depart from its stable operating region.

A number of control approaches have been presented in the literature to address pull-in. In the context of the current paper the most relevant are those that - implicitly or explicitly - make use of the substantial improvements in stability associated with control of electrode charge versus control of electrode voltage. Some notable examples include $[11,15,17]$. An additional challenge is to implement these controllers in the presence of additional resistive and capacitive coupling between device components, and between device components and the surroundings. These interactions are commonly referred to as parasitics. It is known that parasitic capacitance can cause loss of stability of charge-controlled electrostatic MEMS through a saddle-node bifurcation known as charge pull-in $[4,5,13,15]$. Explicit compensation of parasitics is only now beginning to attract attention from the controls community, see for example [18].

In $[6,7,9,10]$ we present a series of results on passivity-based control (PBC) for global and semi-global stabilization of electrostatically actuated MEMS. The one-degree-of-freedom case is considered in [6]. The system input and output are the control voltage applied to the fixed electrode and the charge on the movable electrode, respectively. Two controllers are derived that eliminate the pull-in
\end{abstract}


bifurcation and stabilize any point in the capacitive gap - that is, points between the drive electrode and the zero-voltage equilibrium. Because of the purely attractive capacitively coupled electrostatic forcing, these are the only points for which a feasible equilibrium exists. The first design in [6] is based on the energy-shaping version of PBC (see, for example [14]) and results in a charge feedback controller. The second is based on feedback passivation techniques (first developed in [3]), and requires an additional velocity feedback term. Unlike the energy-shaping controller, the feedback-passivation controller may be used to inject damping into the mechanical subsystem, improving transient performance. The generalized model and controller presented in [7] extends the 1-DOF results to a broad class of electrostatically forced mechanical systems, including a variety of interesting MEMS devices. The extension requires measurement of the voltage and charge associated with all electrodes that influence the movable electrode, including parasitic surfaces. This is typically not practical. The object of the present note is to examine whether the 1-DOF PBC results of [6] may be recovered in the presence of a particular class of parasitics without requiring charge or voltage measurements on the parasitic electrodes.

The model considered is the 1-DOF model used in [6] - a grounded movable electrode suspended by a linear spring and damper above a fixed control electrode - but with an additional fixed parasitic electrode added below the control electrode as shown in Fig. 3.1. Physically the parasitic surface might represent the relatively conductive wafer substrate below the insulating dielectric layer upon which the MEMS device is fabricated. As in $[6,7,1]$, the voltage between the movable and drive electrodes is assumed to be measured, along with the charge on the fixed electrode. Here we will require one additional measurement, namely the charge on the movable electrode. We propose that this quantity be calculated from the voltage across a fixed capacitor placed in series with the movable electrode. Alternately the position of the movable electrode may be measured directly, as with a capacitive bridge. In the present note the parasitic electrode is connected to ground through a resistor. Therefore both the parasitic voltage and the charge can vary dynamically, but at equilibrium the parasitic voltage will be zero. This condition may be relaxed by including a series voltage source, but such an extension is not considered here. 
Texas Tech University, Imiya Manjula Wickramasinghe, May 2008

This note is organized as follows: Section 3.2 presents a 1-DOF model of an electrostatic MEMS with a parasitic capacitance, with two distinct electrode geometries explicitly presented. In Section 3.3 we revisit the PBC results of $[6,7]$ and show how parasitics may cause charge pull-in, which we interpret in terms of a saddle-node bifurcation of the zero dynamics. We then demonstrate how redefining the system output eliminates multiple equilibria of the zero dynamics, thereby preventing charge pull-in for PBC using that output. If the control electrode is larger than either the movable electrode or the parasitic electrode then, neglecting fringing, the parasitic electrode is electrostatically screened from the movable electrode. Section 3.3 shows that in this case the PBC designs may be modified to provide global asymptotic stability of the desired equilibrium. On the other hand, if there is direct capacitive coupling between the movable and parasitic electrodes, then we do not have analytical proof of global asymptotic stability of the closed-loop system using the PBC designs. However, in Section 3.4 we present numerical evidence that the region of attraction of the closed-loop equilibrium point is large, and perhaps global. Additionally Section 3.4 presents simulations of both cases that use the ANSYS finite-element analysis (FEA) package to include fringing fields. For the cases modeled, the presence of these fields affects the accuracy of set-point tracking, but does not destabilize the closed-loop system. Finally, the main contributions are summarized in Section 3.5.

\subsection{A 1-DOF MEMS Model with Parasitic Capacitance}

We consider a MEMS device modeled by three parallel plates, shown schematically in Fig. 3.1. The configuration of the parasitic electrode is motivated by MEMS designs in which a parallel-plate device is surface micromachined on an insulating dielectric layer, which in turn is deposited on a relatively conductive silicon substrate. In such cases large parasitic capacitances may exist between the device and the underlying silicon wafer, especially if the insulating layer is thin. Topmost is the movable electrode, of mass $m$, suspended by linear spring and damping elements, with constants $k$ and $c$, respectively. The movable electrode is constrained to translate in the vertical direction. In the middle is a fixed plate that we refer to as the drive or control electrode, and at the bottom is a fixed parasitic 


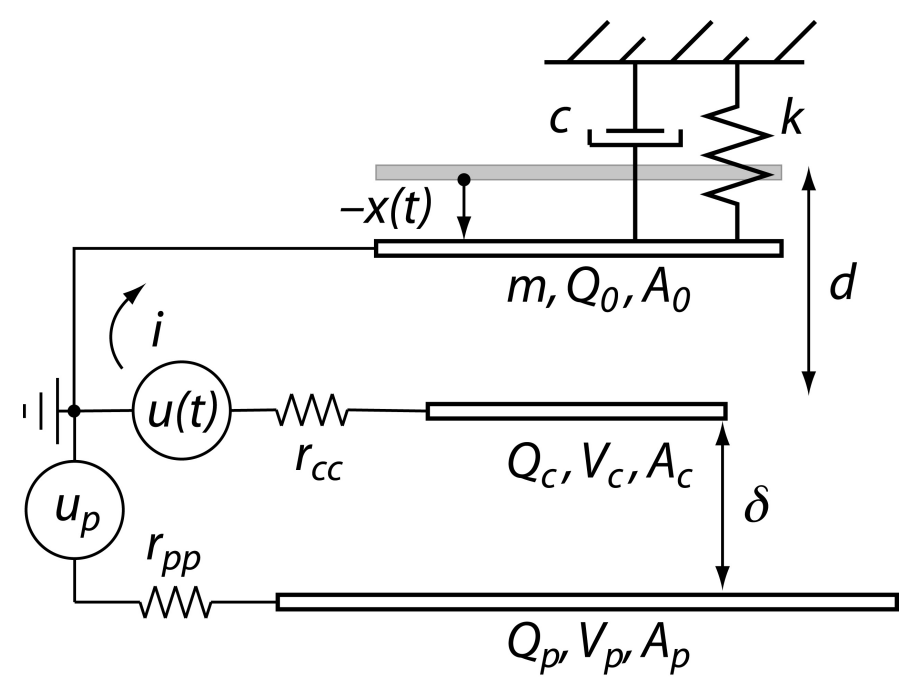

Figure 3.1. 1-DOF model of a electrostatic microactuator. The top plate of the MEMS is free to move and the two bottom plates are held fixed. The middle plate is the control electrode and the bottom plate is the parasitic electrode. This particular configuration is an example of Case II, as defined in the text. The area of the bottom plate may be reduced to that of the top plate without affecting the simple capacitance models used for control design. The parasitic bias voltage $u_{p}$ is assumed to be zero in the controller design and analysis.

electrode. The plates have area $A_{0}, A_{c}$, and $A_{p}$, respectively, and are assumed to be centered on a common axis. The zero-voltage gap between the moving and drive electrode is $d$ and the distance between the parasitic and drive electrode is $\delta$. The displacement of the movable electrode is $x$, defined to be zero in the zero-voltage configuration, and positive in the direction away from the drive electrode. Resistive cross-coupling is neglected, and the movable electrode is assumed to be grounded. A resistance $r_{c c}$ is in series with the drive electrode, voltage source $u(t)$, and movable electrode. A current $i$ flows through this circuit. The charge and voltage on the drive and parasitic electrodes are $Q_{c}, V_{c}$, and $Q_{p}, V_{p}$, respectively. The charge on the movable electrode is $Q_{0}$. The parasitic electrode is connected to ground through a series resistance $r_{p p}$ and bias voltage $u_{p}$. Heceforth $u_{p}$ is assumed to be zero.

For the purpose of control design the capacitive coupling and electrostatic forces are derived using a simple infinite parallel plate model that neglects fringing. The consequences of this assumption will be examined in Section 3.4. Thus the mutual 
capacitance between any two surfaces is modeled as $\tilde{C}_{i j}=\epsilon A_{i j} / l_{i j}$, where $l_{i j}$ is the distance between the surfaces and $A_{i j}$ is the area of the overlap between the surfaces with no intervening conductor. If the parasitic effects are due to interactions with a conductive substrate, this model allows us to consider only that portion of the substrate directly beneath the device. $\epsilon$ is the permittivity of the material in the gap between the plates, assumed here to be constant with value $8.85 \times 10^{-6} \mathrm{pF} / \mu \mathrm{m}$. Capacitances defined with respect to relative potential are designated $\tilde{C}_{i j}$, while elements of the capacitance matrix or its inverse are $C_{i j}$ or $C^{i j}$, respectively.

Even using the simplified 1-DOF model of Fig. 3.1 several distinct electrode configurations are possible. The following observation facilitates the analysis: Under the simplified capacitance calculation, the largest electrode area may, without loss of generality, be reduced to that of the next largest, resulting in three possible situations, in which either the movable electrode is the smallest, the control electrode is the smallest, or the parasitic electrode is the smallest. The first and third situation result in very similar system dynamics, and it suffices to consider one or the other. Since the substrate is larger than the fabricated device, the first case is of greater practical interest. Therefore, to avoid unnecessarily complex notation, we will consider only the first and second configurations, which we subsequently refer to as Case I and Case II. We define the parameter $\rho=A_{0} / A_{c}$. For Case I, $\rho \leq 1$ and for Case II $\rho>1$. In Case I the movable electrode is completely screened from the parasitic electrode by the control electrode. In Case II the movable electrode is directly affected by the parasitics.

Application of the modeling procedure presented in [7], gives the following equations of motion:

$$
\begin{aligned}
\dot{Q}_{c} & =-\frac{1}{r_{c c}} V_{c}+\frac{1}{r_{c c}} u_{c} \\
& =-\frac{1}{r_{c c}} C^{c p}(x)\left(\alpha^{c c}(x) Q_{c}+Q_{p}\right)+\frac{1}{r_{c c}} u_{c} \\
\dot{Q}_{p} & =-\frac{1}{r_{p p}} C^{c p}(x)\left(Q_{c}+\alpha^{p p}(x) Q_{p}\right), \\
\dot{x} & =v \\
\dot{v} & =-2 \zeta \omega v-\omega^{2} x-f^{e}\left(x, Q_{c}, Q_{p}\right),
\end{aligned}
$$


where the electrostatic force term $f^{e}\left(x, Q_{c}, Q_{p}\right)$ is of the form

$$
f^{e}\left(x, Q_{c}, Q_{p}\right)=\frac{1}{2 m \epsilon A_{0}}\left(f_{11}^{e}(x) Q_{c}^{2}+2 Q_{c} Q_{p}+Q_{p}^{2}\right)
$$

Here $Q_{c}$ and $Q_{p}$ are the charge on the control and parasitic electrode, respectively. $V_{c}$ is the voltage on the control electrode, $u_{c}$ is the voltage applied at the controller, and $x$ and $v$ are the displacement and velocity, respectively, of the movable electrode. The mechanical subsystem is characterized by natural frequency $\omega=\sqrt{k / m}$ and damping ratio $\zeta=c / 2 m \omega$, respectively. As seen in Fig. 3.1, $r_{c c}$ and $r_{p p}$ are resistances in series with the control and parasitic electrodes, respectively.

Charge and voltage are related by

$$
\left[\begin{array}{l}
V_{c} \\
V_{p}
\end{array}\right]=C^{c p}(x)\left[\begin{array}{cc}
\alpha^{c c}(x) & 1 \\
1 & \alpha^{p p}(x)
\end{array}\right]\left[\begin{array}{l}
Q_{c} \\
Q_{p}
\end{array}\right]
$$

Equation (3.7) defines the functions $C^{c p}(x), \alpha^{c c}(x)$, and $\alpha^{p p}(x)$, which have no other explicit physical meaning. Likewise (3.6) defines $f_{11}^{e}$. Table 3.1 lists values for these functions in the two electrode geometry cases.

\subsection{Passivity-Based Control Design and Analysis Incorporating Parasitics}

The nonlinear phenomenon of voltage pull-in associated with voltage control of electrostatic MEMS is well known. Details may be found, for example, in [16]. A number of researchers demonstrate controllers based on electrode charge to eliminate voltage pull-in in the absence of parasitics $[11,15]$. Similarly, we have previously shown for the 1-DOF MEMS without parasitics that the choice of control electrode charge as the system output in the PBC framework causes the zero dynamics to have a unique equilibrium point, and that static or dynamic output feedback may be used to make that equilibrium point a global attractor $[6,7]$. In the presence of parasitics those previous results no longer hold.

With the parasitic plate, the equation governing the equilibrium position of the electrode with the output $y=Q_{c}-\bar{Q}_{c} \equiv 0$ is for Case I, $-\bar{x}(\bar{x}+d+\rho \delta)^{2}=\rho^{2} \delta^{2} \bar{Q}_{c}^{2} / 2 \epsilon A_{0} m \omega^{2}$, and for Case II, $-\bar{x}(\bar{x}+d+\delta)^{2}=\rho \delta^{2} \bar{Q}_{c}^{2} / 2 \epsilon A_{0} m \omega^{2}$. In these equations and subsequently an overbar 
Texas Tech University, Imiya Manjula Wickramasinghe, May 2008

Table 3.1. Function values for the two distinct electrode geometries. $\rho \equiv A_{0} / A_{c}$. $d$ is the zero-voltage gap. $\tilde{C}_{c c}$ is the capacitance between the control electrode and the grounded movable electrode. The term $C^{c p}$ arises from the inverse capacitance matrix. It is included here for completeness even though it is the same for both cases.

\begin{tabular}{|c|c|c|}
\hline Case & I & II \\
\hline \hline Smallest Area & $A_{0}$ & $A_{c}$ \\
\hline Other Areas & $A_{c}=A_{p}$ & $A_{0}=A_{p}$ \\
\hline$f_{11}^{e}$ & 1 & $1+\frac{\delta^{2}(\rho-1)}{(x+d+\delta)^{2}}$ \\
\hline$\alpha^{c c}$ & 1 & $1+\frac{\delta(\rho-1)}{x+d+\delta}$ \\
\hline$\alpha^{p p}$ & $1+\frac{\rho \delta}{(x+d)}$ & $1+\frac{\delta}{x+d}$ \\
\hline$\tilde{C}_{c c}(x)$ & $\epsilon A_{0} /(x+d)$ & $\epsilon A_{0} / \rho(x+d)$ \\
\hline$C^{c p}(x)$ & $(x+d) / \epsilon A_{0}$ & $(x+d) / \epsilon A_{0}$ \\
\hline
\end{tabular}

implies an equilibrium value. Specifically, $\bar{Q}_{c}$ is the desired equilibrium value of $Q_{c}$ that is used in the output equation, and $\bar{x}$ is the resulting equilibrium value of $x$. The appropriate $\bar{Q}_{c}$ must be calculated and input to the controller. Both these cases may be put in the form $-x^{*}\left\{\mu_{I, I I}\left(x^{*}+1\right)+1\right\}^{2}=\gamma_{I, I I}$, where $x^{*}=\bar{x} / d$. For Case I, $\mu_{I}=d / \rho \delta, \gamma_{I}=\bar{Q}_{c}^{2} / 2 \epsilon A_{0} m \omega^{2} d$, and for Case II, $\mu_{I I}=d / \delta, \gamma_{I I}=\rho \bar{Q}_{c}^{2} / 2 \epsilon A_{0} m \omega^{2} d$. Investigating these equilibrium equations we find that a new saddle-node bifurcation, referred to as "charge pull-in" $[4,5,13,15]$, occurs when $\mu_{I, I I} \geq 1 / 2$. Charge pull-in occurs at $\bar{x}=-(d / 3)-(\rho \delta / 3)$ or $-(d / 3)-(\delta / 3)$ for Case I or II, respectively. Figure 3.2 shows this behavior graphically as the parameter $\mu_{I, I I}$ varies. The contribution of this note is to show how the controllers of [6] may be modified to recover their nominal stability and performance characteristics in the presence of parasitics.

In the absence of parasitics, the choice of charge instead of voltage as the output in a passivity framework eliminates voltage pull-in, so it is natural to ask whether there is another choice of output for which, in the presence of parasitics, eliminates charge pull-in. To investigate this question we introduce a new output 


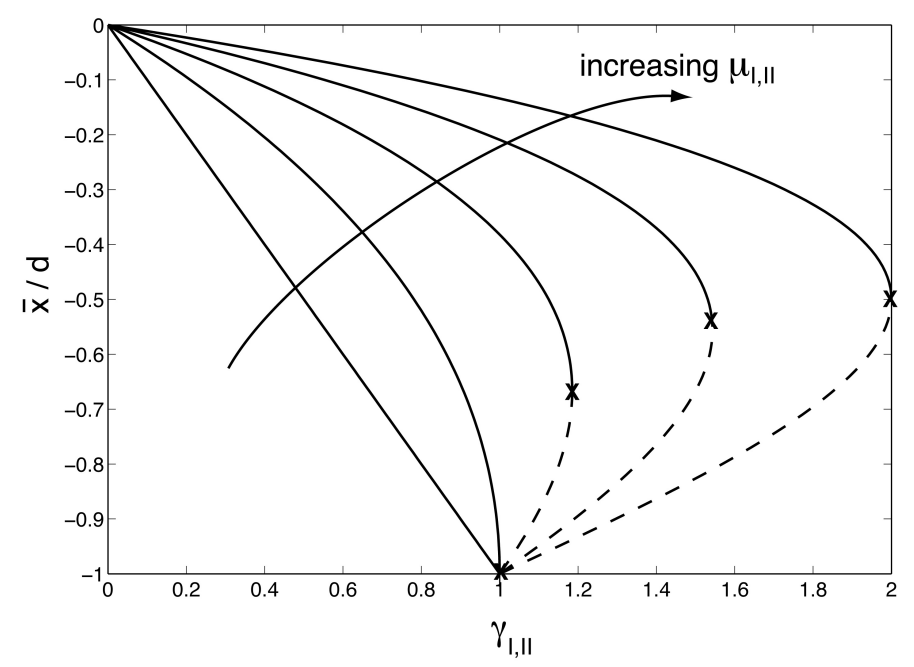

Figure 3.2. Bifurcation diagrams for the equilibrium curves $-x^{*}\left\{\mu_{I, I I}\left(x^{*}+1\right)+1\right\}^{2}=$ $\gamma_{I, I I}$ of the zero dynamics of a 1-DOF MEMS model under charge control in the presence of parasitics, as parameter $\mu_{I, I I}$ varies. Curves correspond to $\mu_{I, I I}=0,0.5$, $1,1.5$, and 2. For $\mu_{I, I I} \geq 0.5$, charge pull-in occurs at the points marked by an 'x.' Stable branches are solid lines, unstable branches are dashed. For Case I, $\mu_{I}=d / \rho \delta$ and $\gamma_{I}=\bar{Q}_{c}^{2} / 2 \epsilon A_{0} m \omega^{2} d$. For Case II, $\mu_{I I}=d / \delta$ and $\gamma_{I I}=\rho \bar{Q}_{c}{ }^{2} / 2 \epsilon A_{0} m \omega^{2} d$.

$y=\tilde{Q}_{c c}-\overline{\tilde{Q}}_{c c}$, where $\tilde{Q}_{c c}$ is defined to be the portion of the charge on the control electrode specifically induced by $\tilde{C}_{c c}(x)$, the mutual capacitance between the movable electrode and the control electrode. $\overline{\tilde{Q}}_{c c}$ is the desired equilibrium value of $\tilde{Q}_{c c}$. The appropriate $\overline{\tilde{Q}}_{c c}$ must be calculated and input to the controller.

$$
\begin{aligned}
\tilde{Q}_{c c} & =\tilde{C}_{c c}(x) V_{c}(x) \\
& =\tilde{C}_{c c}(x) C^{c p}(x)\left(\alpha^{c c}(x) Q_{c}+Q_{p}\right)
\end{aligned}
$$

Table 3.2 summarizes the equilibrium values for the electrostatic force and the zero dynamics states $x$ and $Q_{p}$ in terms of the output set point $\overline{\tilde{Q}}_{c c}$. As can be seen from Table 3.2, for both Case I and Case II, a given $\overline{\tilde{Q}}_{c c}$ corresponds to a unique $\bar{x}$. This proves the uniqueness of the equilibrium of the zero dynamics, and thereby shows that charge pull-in is eliminated. Other output functions also have this property; this function was selected based on the large region of attraction seen in simulation of the corresponding passivity-based controllers for Case II geometries, as 
Table 3.2. Zero dynamics equilibrium conditions with output $y=\tilde{Q}_{c c}-\overline{\tilde{Q}}_{c c}$.

\begin{tabular}{|c|c|c|}
\hline Case & I & II \\
\hline \hline $\bar{Q}_{p}$ & $-\frac{(\bar{x}+d)}{\rho \delta} \overline{\tilde{Q}}_{c c}$ & $-\frac{(\bar{x}+d)}{\delta} \overline{\tilde{Q}}_{c c}$ \\
\hline$f^{e}\left(\bar{x}, \overline{\tilde{Q}}_{c c}, \bar{Q}_{p}\right)$ & $\frac{\tilde{\tilde{Q}}_{c c}^{2}}{2 m \epsilon A_{0}}$ & $\frac{\rho \tilde{Q}_{c c}^{2}}{2 m \epsilon A_{0}}$ \\
\hline $\bar{x}$ & $-\frac{\bar{Q}_{c c}^{2}}{2 m \epsilon A_{0} \omega^{2}}$ & $-\frac{\rho \tilde{\tilde{Q}}_{c c}^{2}}{2 m \epsilon A_{0} \omega^{2}}$ \\
\hline
\end{tabular}

discussed further below.

We now show that for Case I the static and dynamic controllers presented in [6] using output $y=Q_{c}-\bar{Q}_{c}$ globally asymptotically stabilize the equilibrium given in Table 3.2 when the output is instead $y=\tilde{Q}_{c c}-\overline{\tilde{Q}}_{c c}$. Applying $\overline{\tilde{Q}}_{c c}=\sqrt{-2 m \epsilon A_{0} \omega^{2} \bar{x}}$ gives the value of $\overline{\tilde{Q}}_{c c}$ corresponding to the desired gap $\bar{x}$.

Substituting Case I values for $\tilde{C}_{c c}(x), C^{c p}(x)$ and $\alpha^{c c}(x)$ from Table 3.1 into (3.9) yields $\tilde{Q}_{c c}=Q_{c}+Q_{p}$. Rewrite the state equations (3.1)-(3.5) using $\tilde{Q}_{c c}$ as a state instead of $Q_{c}$, and apply the input-output linearizing control

$$
\begin{aligned}
u_{c}=r_{c c} & \left(\left(\frac{1}{r_{c c}}+\frac{\alpha^{p p}(x)}{r_{p p}}\right) V_{c}+\right. \\
& \left.\frac{C^{c p}(x)\left(1-\alpha^{c c}(x) \alpha^{p p}(x)\right)}{r_{p p}} Q_{c}+\nu\right) .
\end{aligned}
$$

As in [6], implementation of this feedback control requires $V_{c}, Q_{c}$, and $x$, which for the moment we assume are known. We obtain

$$
\begin{aligned}
\dot{\tilde{Q}}_{c c} & =\nu \\
\dot{x} & =v \\
\dot{v} & =-2 \zeta \omega v-\omega^{2} x-f^{e}\left(x, \tilde{Q}_{c c}\right), \\
\dot{Q}_{p} & =-\frac{C^{c p}(x)}{r_{p p}}\left(\left(\alpha^{p p}(x)-1\right) Q_{p}+\tilde{Q}_{c c}\right),
\end{aligned}
$$


where

$$
f^{e}\left(x, \tilde{Q}_{c c}\right)=\frac{1}{2 m \epsilon A_{0}} \tilde{Q}_{c c}^{2}
$$

We see that subsystem (3.11)-(3.13) is uncoupled from the parasitic subsystem (3.14), and is identical to the system considered in [6] with $Q_{c}$ replaced by $\tilde{Q}_{c c}$. Thus, by the proofs presented in that paper, the static output feedback controller

$$
\nu=-k\left(\tilde{Q}_{c c}-\overline{\tilde{Q}}_{c c}\right)
$$

with $k>0$ is globally asymptotically stabilizing for this system as well.

Implementation of (3.15) requires $\tilde{Q}_{c c}$. From (3.8) we see this can be implemented using measurements $V_{c}$ and $x$, and a model of the capacitance $\tilde{C}_{c c}(x)$. Similarly, the dynamic output feedback controller given by [6],

$$
\nu=\frac{1}{2 \epsilon A_{c}}\left(\tilde{Q}_{c c}+\overline{\tilde{Q}}_{c c}\right) v-k\left(\tilde{Q}_{c c}-\overline{\tilde{Q}}_{c c}\right)
$$

is also globally asymptotically stabilizing with $k>0$. The dynamic compensator is preferred over the static one when additional damping is desired for performance reasons [6]. In (3.16) it is required that the movable electrode velocity $v$ be known, which is typically unrealistic. Therefore a dynamic observer is needed to estimate $v$. With $\tilde{Q}_{c c}$ and $x$ measured, the system has the structure $\dot{x}=A x+f(y), y=C x$. That is, the nonlinearity is a function only of the measurement $y$, and $y$ is a linear combination of the states. The full-order observer takes the form

$$
\begin{aligned}
{\left[\begin{array}{c}
\dot{\hat{\tilde{Q}}} \\
\dot{\hat{x}} \\
\dot{\hat{v}}
\end{array}\right] } & =\left[\begin{array}{ccc}
0 & 0 & 0 \\
0 & 0 & 1 \\
0 & -\omega^{2} & -2 \zeta \omega
\end{array}\right]\left[\begin{array}{c}
\hat{\tilde{Q}}_{c c} \\
\hat{x} \\
\hat{v}
\end{array}\right] \\
& +\left[\begin{array}{c}
0 \\
0 \\
-\tilde{Q}_{c c}^{2}
\end{array}\right]+\left[\begin{array}{l}
1 \\
0 \\
2 m \epsilon A_{0}
\end{array}\right] \nu \\
& +L\left(\left[\begin{array}{c}
\tilde{Q}_{c c} \\
x
\end{array}\right]-\left[\begin{array}{c}
\hat{\tilde{Q}}_{c c} \\
\hat{x}
\end{array}\right]\right)
\end{aligned}
$$


Proof of stability of the closed-loop subsystem (3.11)-(3.13) and (3.17) exactly follows [6].

The parasitic dynamics (3.14) are

$$
\dot{Q}_{p}=-\frac{\rho \delta}{r_{p p} \epsilon A_{0}} Q_{p}-\frac{x+d}{r_{p p} \epsilon A_{0}} \tilde{Q}_{c c}
$$

which, due to the decoupling and asymptotic stability of the rest of the system, may be treated as a linear, exponentially stable, first-order equation with a bounded and convergent exogenous input. Such a system must converge to a steady value. Thus for Case I, either (3.15) or (3.16) globally asymptotically stabilizes the system corresponding to any $\overline{\tilde{Q}}_{c c}$.

This concludes the discussion of stability for Case I. In Case II the parasitic dynamics are not decoupled by the change of coordinates from $Q_{c}$ to $\tilde{Q}_{c c}$. Despite the lack of analytical proof of convergence, in the next section we apply the same two controllers (3.15) and (3.16), and numerically examine the result. We see that the simulated performance is very promising.

We have assumed that $V_{c}, Q_{c}$, and $x$ are measured, or may be inferred from measurements. It is reasonable to make voltage measurements on engineered components such as the control and movable electrodes. As presented in $[6,7,1]$, by measuring the voltage across fixed capacitors placed in series with these components one can also obtain their charge. If charge and voltage measurements are available for all electrodes then the capacitance models can in principle be inverted to find the displacement $x$. In the case with parasitics the voltage of the parasitic surface is not known. However it is still possible, in principle, to find $x$ using capacitance models from measurements $V_{c}, Q_{c}$, and $Q_{0}$ through eliminating $V_{p}$ from the equations $Q_{c}=\left(\tilde{C}_{c c}(x)+\tilde{C}_{c p}\right) V_{c}+\tilde{C}_{c p} V_{p}$ and $Q_{0}=-\tilde{C}_{c c}(x) V_{c}-\tilde{C}_{p p}(x) V_{p}$. Although this method does not require measurement of $V_{p}$, it does requires a parasitic capacitance model $\tilde{C}_{c p}$ and $\tilde{C}_{p p}(x)$. Alternately, one may directly measure $x$ using a capacitive bridge or other means. 


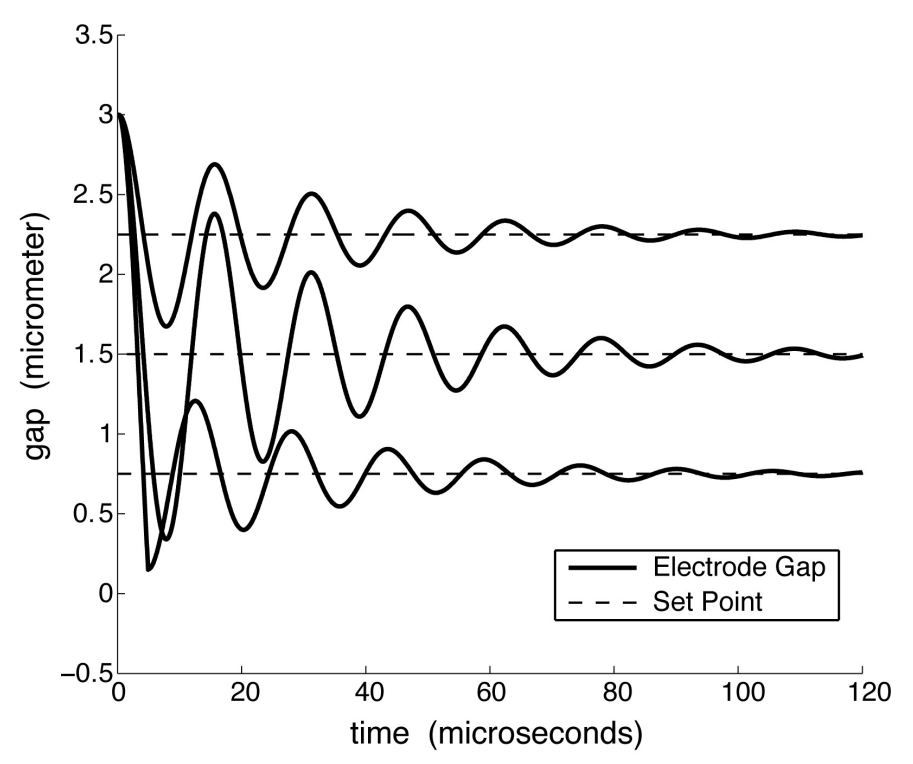

Figure 3.3. Simulation results for Case II with static output feedback for three values of $\bar{x}$, with $\delta=0.5$ and $\rho=3$.

\subsection{Simulation Results}

This section presents representative results from a $M A T L A B$ simulation study of Case II, showing stability of the zero dynamics and closed-loop system over a range of parameters and initial conditions. First the nominal performance is examined, using parallel plate capacitance models in both the simulation equations and the controller. Next we examine the effects of this assumption on performance, through simulations for Cases I and II using capacitances and forces computed using the ANSYS Multiphysics finite-element package.

\subsubsection{Stability and Domain of Attraction for Case II}

Given detectability, stability of the closed-loop system under PBC depends on the stability of the zero dynamics. We have examined the poles of the linearized zero dynamics of (3.1)-(3.6) for Case II, with $\tilde{Q}_{c c}-\overline{\tilde{Q}}_{c c}$ as output, as $\bar{x}$ varies from 0 to $-d$ for $\delta=0.001$ and $\rho=5$. A real pole moves by only about $1.5 \%$, from about $-2.82 \times 10^{4}$ to about $-2.78 \times 10^{4}$. A complex conjugate pair of poles corresponding to the dynamics of the mechanical subsystem does not move at all. The location of 


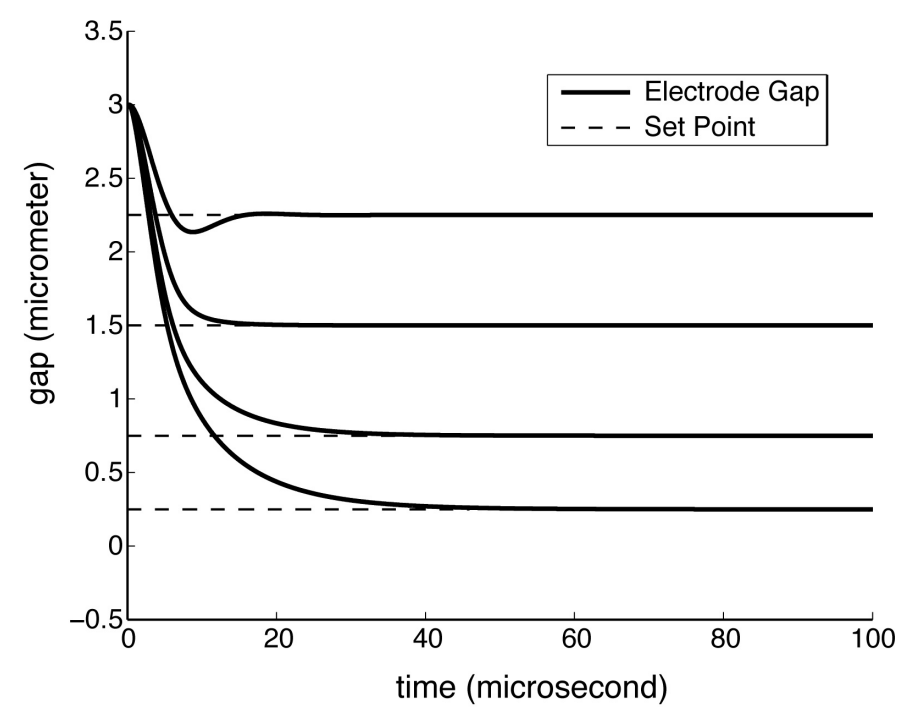

Figure 3.4. Simulation results for Case II with dynamic output feedback for four values of $\bar{x}$, with $\delta=0.5$ and $\rho=3$.

this pair is determined only by the parameters $\omega$ and $\zeta$ in (3.5) and is unaffected by the $\bar{x}$ setpoint. For the parameter values studied they are at about $-3.47 \times 10^{4} \pm i 4.02 \times 10^{5}$. Similar results are found for a range of parasitic geometries with $0.001 \leq \delta \leq 2,1 \leq \rho \leq 10$. The location of the complex conjugate pair does not change, and the real root remains in the open left half-plane for all parameter values studied. Thus the closed-loop system will be locally asymptotically stable for any feasible equilibrium over a wide range of parasitic electrode configurations.

We will apply the same state-feedback controllers (3.15) and (3.16) to Case II that have previously been applied to Case I. However the implemented dynamic output feedback controller must be modified, because the observer design differs between 
the cases. Separating the linear and nonlinear terms in Case II gives the form

$$
\begin{aligned}
{\left[\begin{array}{c}
\dot{\tilde{Q}}_{c c} \\
\dot{x} \\
\dot{v}
\end{array}\right] } & =\left[\begin{array}{ccc}
0 & 0 & 0 \\
0 & 0 & 1 \\
0 & -\omega^{2} & -2 \zeta \omega
\end{array}\right]\left[\begin{array}{c}
\tilde{Q}_{c c} \\
x \\
v
\end{array}\right] \\
+ & {\left[\begin{array}{c}
0 \\
0 \\
-f^{e}\left(x, Q_{c}, Q_{p}\right)
\end{array}\right]+\left[\begin{array}{l}
1 \\
0 \\
0
\end{array}\right] \nu }
\end{aligned}
$$

Here $f^{e}\left(x, Q_{c}, Q_{p}\right)$ is given by

$$
f^{e}\left(x, Q_{c}, Q_{p}\right)=\frac{1}{2 m \epsilon A_{0}}\left(f_{11}^{e}(x) Q_{c}^{2}+2 Q_{c} Q_{p}+Q_{p}^{2}\right)
$$

This is apparently not of the desired form $\dot{x}=A x+f(y)$, since $f^{e}\left(x, Q_{c}, Q_{p}\right)$ contains $Q_{p}$, which is not a measurement. However, $Q_{p}$ can be written as

$$
Q_{p}\left(x, V_{c}, Q_{c}\right)=\frac{V_{c}}{C^{c p}(x)}-\alpha^{c c}(x) Q_{c}
$$

Therefore we have the following full-order observer:

$$
\begin{aligned}
{\left[\begin{array}{c}
\dot{\tilde{\tilde{Q}}}_{c c} \\
\dot{\hat{x}} \\
\dot{\hat{v}}
\end{array}\right] } & =\left[\begin{array}{ccc}
0 & 0 & 0 \\
0 & 0 & 1 \\
0 & -\omega^{2} & -2 \zeta \omega
\end{array}\right]\left[\begin{array}{c}
\hat{\tilde{Q}}_{c c} \\
\hat{x} \\
\hat{v}
\end{array}\right] \\
& +\left[\begin{array}{c}
0 \\
-f^{e}\left(x, Q_{c}, Q_{p}\left(x, V_{c}, Q_{c}\right)\right)
\end{array}\right]+\left[\begin{array}{l}
1 \\
0 \\
0
\end{array}\right] \nu \\
& +L\left(\left[\begin{array}{c}
\tilde{Q}_{c c} \\
x
\end{array}\right]-\left[\begin{array}{c}
\hat{\tilde{Q}}_{c c} \\
\hat{x}
\end{array}\right]\right)
\end{aligned}
$$

with $f^{e}\left(x, Q_{c}, Q_{p}\left(x, V_{c}, Q_{c}\right)\right)$ given by (3.19).

We perform MATLAB simulations of Case II implementing (3.1)-(3.6), with 
output $y=\tilde{Q}_{c c}-\overline{\tilde{Q}}_{c c}$. given by (3.9). For the purpose of studying nominal performance, the capacitances and forces in these equations are initially assumed to be the same parallel plate approximations used in the controller. This assumption is lifted in the next section. The feedback linearizing term of the controller is given by (3.10). The stabilizing term $\nu$ is given by (3.15) for the static controller, and (3.16) for the dynamic controller. The velocity observer is given by(3.20). System parameters are chosen to be $\omega=4.0397 \times 10^{5}, \zeta=0.086, d=3, r_{c c}=r_{p p}=10^{-6}$. For both the static and dynamic controllers the controller gain is set to $k=10^{7}$. For the dynamic controller the observer gain $L$ is chosen to place the poles of the linear error dynamics at $\left\{-3.0 \times 10^{7},-3.1 \times 10^{7},-3.2 \times 10^{7}\right\}$. The parasitic plate geometry parameters were set to $\rho=3$ and $\delta=0.5$. The target setpoints $\bar{x}$, are listed separately in the figures showing the results of each simulation.

Figures 3.3 and 3.4 show typical simulation results for the static and dynamic controllers, respectively. The simulations show stable transitions from $\bar{x}=0$ to a range of final target values. As expected, dynamic output feedback controller has improved transient performance, while the static output feedback controller is limited by the open-loop dynamics of the mechanical subsystem. Similar results are found for a range of parasitic geometries with $0.001 \leq \delta \leq 2,1 \leq \rho \leq 10$. We believe that these ranges cover most actual MEMS devices.

The simulation results reported here show that the PBC designs with system output $\tilde{Q}_{c c}$ are asymptotically stabilizing over a wide range of parasitic geometries and initial conditions. No unstable behavior was seen, leading us to speculate that these controllers may be globally asymptotically stabilizing for all parameter values for Case II as well as for Case I. This result is highly dependent on the choice of output functions. Other choices eliminate charge pull-in, but are not stable over as large a range of parameters and initial conditions. An example may be seen in [10], where the output function considered is the total charge $Q_{c}+Q_{p}$ (or equivalently, the charge on the movable electrode $Q_{0}$ ). There it is seen that although the pull-in bifurcation does not occur, the system can still lose stability for setpoints low in the gap through a subcritical Hopf bifurcation. 


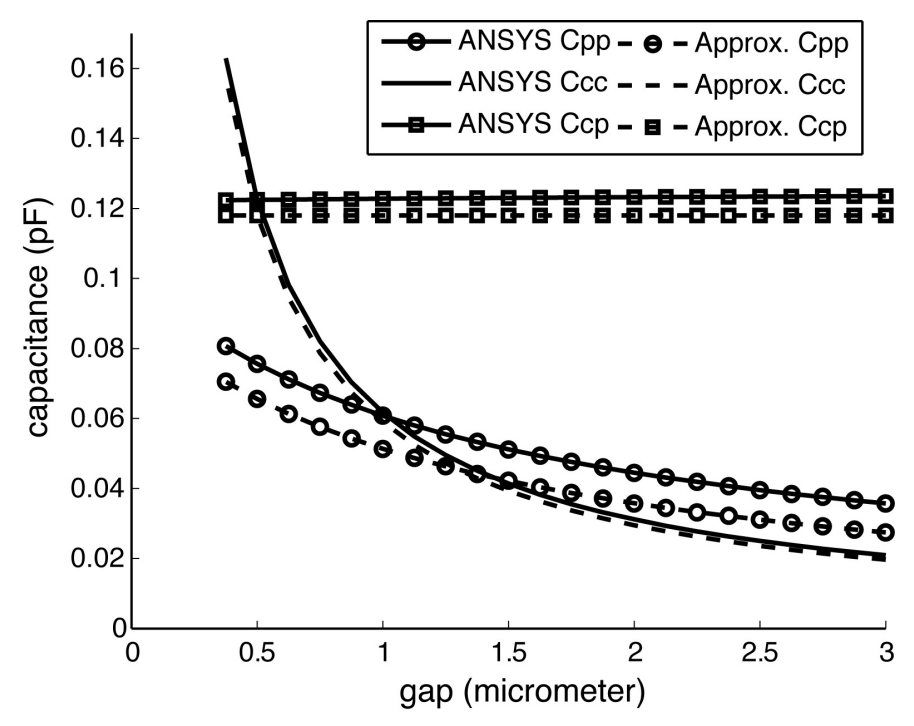

Figure 3.5. Comparison of parallel plate capacitance approximations to capacitances computed using ANSYS. The movable electrode is $100 \mu \mathrm{m}$ by $200 \mu \mathrm{m}$, the control electrode is $57.7 \mu \mathrm{m}$ by $115.5 \mu \mathrm{m}$, and the parasitic electrode is $135 \mu \mathrm{m}$ by $270 \mu \mathrm{m}$, corresponding to $\rho=3$. The parasitic plate spacing $\delta$ is 0.5 .

\subsubsection{Simulation of Cases I and II with Modeling Errors}

In this section we examine the impact of the parallel plate capacitance approximation on controller performance. To reflect the interpretation of the parasitic electrode as the conductive substrate, the parasitic electrode is chosen to be larger than the other two surfaces (exact values are specified in the figures describing each simulation result). Other values are as in the MATLAB simulations described in the previous section.

The ANSYS Multiphysics finite-element analysis package allows calculation of the electrostatic field between multiple conductors, including fringing fields, through the solution of Laplace's equation [2]. ANSYS Multiphysics includes a utility called CMATRIX for computing capacitances based on these solutions. It is possible to use ANSYS directly for dynamic simulation, as was done in [1]. However, for a $1-D O F$ system this is computationally wasteful, as the fields are recalculated multiple times for the same configuration. Instead, capacitances were computed over a range of electrode gap values. An interpolation function was used with these 


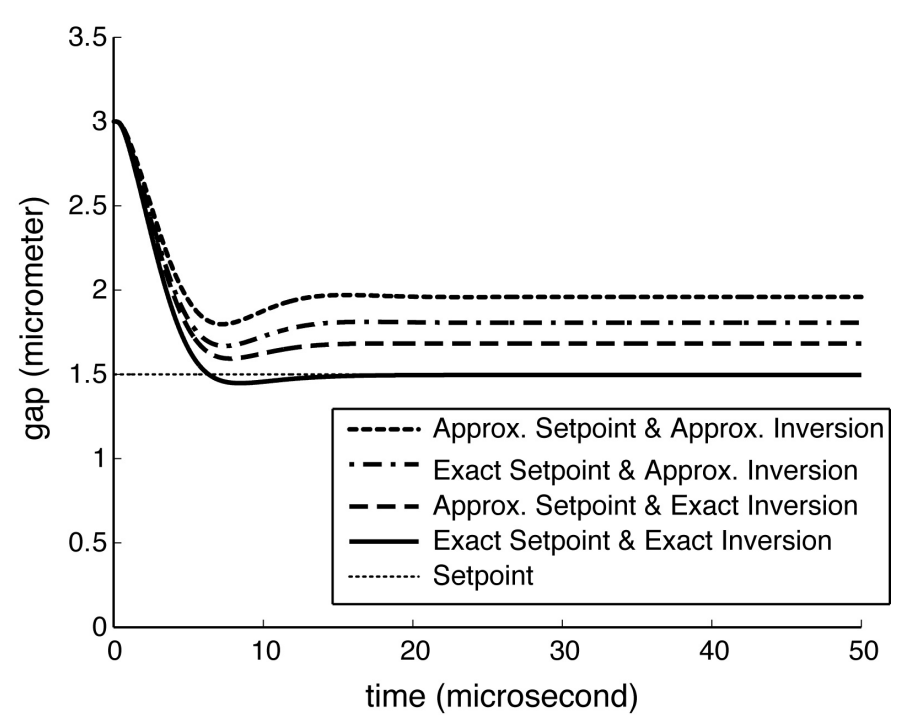

Figure 3.6. Simulation of dynamic output feedback control of Case II, comparing use of the parallel plate approximation to use of capacitances and forces computed using ANSYS. The movable electrode is $100 \mu \mathrm{m}$ by $200 \mu \mathrm{m}$, the control electrode is 57.7 $\mu \mathrm{m}$ by $115.5 \mu \mathrm{m}$, and the parasitic electrode is $135 \mu \mathrm{m}$ by $270 \mu \mathrm{m}$, corresponding to $\rho=3$.

data to generate realistic capacitances at any gap. The electrostatic forces on the movable electrode can then be computed using the formula $f^{e}=(1 / 2)(\partial C / \partial x) V^{2}$. The force must be normalized by the mass of the movable electrode, which is taken to be $4.32 \times 10^{-11} \mathrm{~kg}$. These values are used in in a MATLAB simulation of (3.1)-(3.5). Figure 3.5 compares the parallel plate approximation to ANSYS results for a particular electrode configuration at varying gap widths.

The simplified parallel plate capacitance models are used twice in the controller derivation, first to obtain the $\overline{\tilde{Q}}_{c c}$ that will give a desired position $\bar{x}$, and then implicitly in the linearizing feedback (3.10). To determine the effect of each of these on the controller, simulations were performed of dynamic output feedback control of Case II with $\rho=3$ and $\delta=0.5$ using $A N S Y S$ and parallel plate models for each of these terms, both separately and together. Since (3.1)-(3.5) use the ANSYS values, there is no modeling error when these are used in the controller. Figure 3.6 shows the results of four simulations. In the first, the parallel plate approximation is used both to compute $\overline{\tilde{Q}}_{c c}$ and to input-output linearize the system. In the second 
$A N S Y S$ values are used to compute the setpoint while the parallel plate model is used for for linearization. The third uses the parallel plate approximations to compute $\overline{\tilde{Q}}_{c c}$, while the linearizing feedback is generated using the ANSYS values. Finally, $A N S Y S$ values are used for both purposes. The results show that an error of about $20 \%$ is associated with use of approximate values in the linearizing feedback, while an error of about $12 \%$ is associated with the imperfect calculation of $\overline{\tilde{Q}}_{c c}$. Together these give a setpoint error of almost $30 \%$. This offset is considerably larger that the difference between the parallel plate and $A N S Y S$ models themselves. Addressing this error is a topic for future work, but we note that the more accurate finite-element curves may be incorporated into the actual controller to provide improved performance by substituting them into the appropriate controller formulas.

Figures 3.7-3.9 show similar results for Case I with static output feedback, Case I with dynamic output feedback, and Case II with static output feedback, respectively. In each plot two simulations are shown, one using the parallel plate approximation to both compute $\overline{\tilde{Q}}_{c c}$ and input-output linearize the system, the other using ANSYS values for these purposes. The results also show offsets due to the simplified capacitance models as seen above. However the response is stable in every case, and use of the more precise capacitance curves recovers the desired accuracy.

\subsection{Conclusion}

This chapter presents a 1-DOF model including a capacitively coupled parasitic electrode, specialized from a general modeling and controls framework for electrostatically actuated MEMS. Previous work by the authors presented static and dynamic output feedback controllers that, in the absence of parasitics, globally asymptoticallystabilize any equilibrium electrode configuration, thereby eliminating voltage pull-in and allowing the movable electrode an extended travel range. These controllers were based on the observation that the bifurcation that occurs in the zero dynamics when drive electrode voltage is chosen as the system output does not occur when drive electrode charge is chosen instead. However a sufficiently large parasitic capacitance will destroy this property, through a saddle-node bifurcation known in the literature as charge pull-in. This again limits the operational range of travel of the movable electrode, though less severely than voltage pull-in. For the 


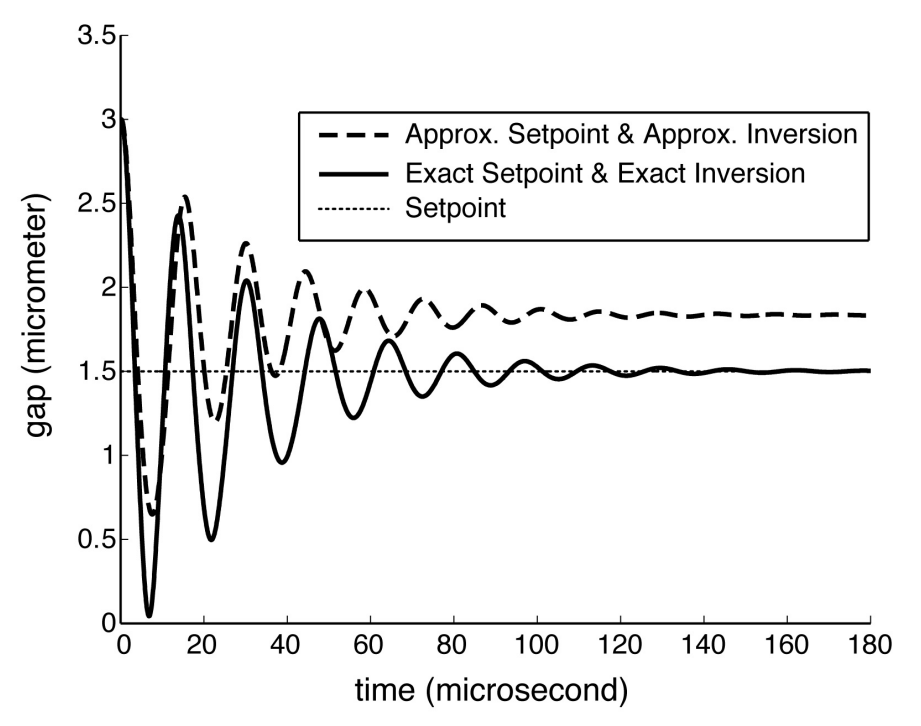

Figure 3.7. Simulation of static output feedback control of Case I, comparing the parallel plate approximation to capacitances computed using ANSYS. The movable electrode is $100 \mu \mathrm{m}$ by $200 \mu \mathrm{m}$, the control electrode is $112 \mu \mathrm{m}$ by $223 \mu \mathrm{m}$, and the parasitic electrode is $162.4 \mu \mathrm{m}$ by $323.4 \mu \mathrm{m}$, corresponding to $\rho=0.8$.

1-DOF model considered, we showed how the choice of a different quantity, denoted $\tilde{Q}_{c c}$, as the system output eliminates the charge pull-in bifurcation. Two qualitatively different electrode geometries must be considered. For the first, we recover analytically the global asymptotic stability result obtained without parasitics.In the second, we rely on numerical simulation results to show that the domain of attraction of the controllers is large. Finite-element simulations show that the parallel plate approximation used in controller design may introduce significant offsets in the steady state position of the movable electrode, with most of this due to the dynamic inversion component. This effect was reduced in simulation by using more accurate capacitance models. 


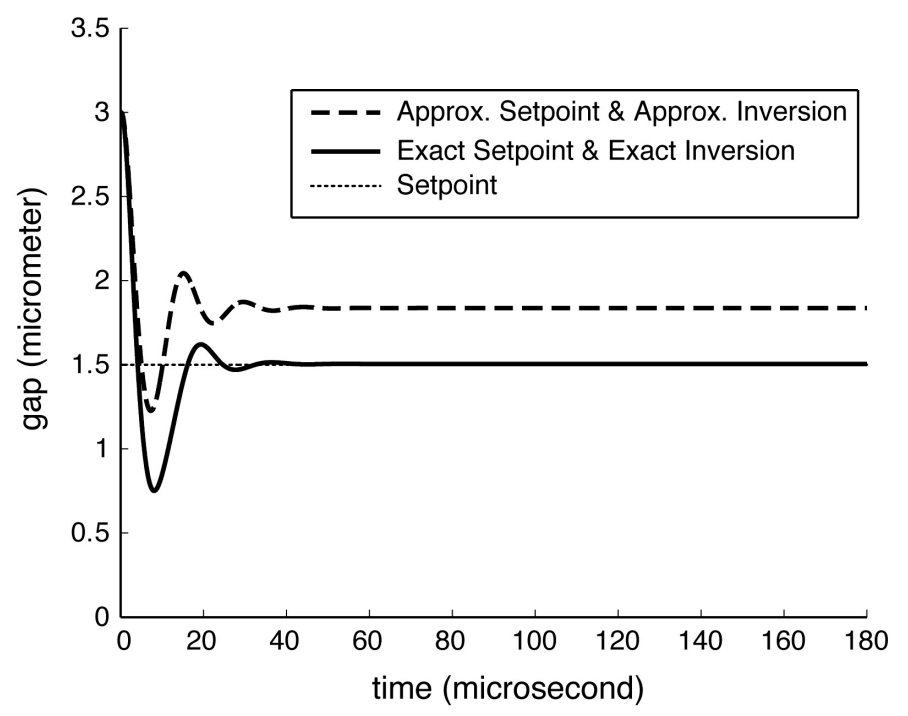

Figure 3.8. Simulation of dynamic output feedback control of Case I, comparing the parallel plate approximation to capacitances computed using ANSYS. The movable electrode is $100 \mu \mathrm{m}$ by $200 \mu \mathrm{m}$, the control electrode is $112 \mu \mathrm{m}$ by $223 \mu \mathrm{m}$, and the parasitic electrode is $162.4 \mu \mathrm{m}$ by $323.4 \mu \mathrm{m}$, corresponding to $\rho=0.8$.

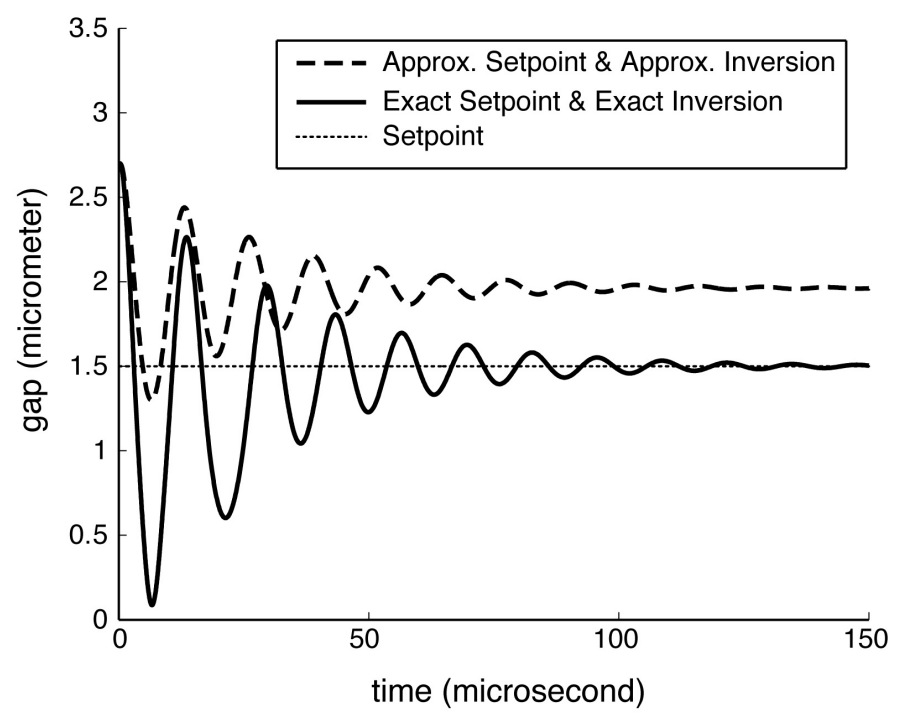

Figure 3.9. Simulation of static output feedback control of Case II, comparing the parallel plate approximation to capacitances computed using ANSYS. The movable electrode is $100 \mu \mathrm{m}$ by $200 \mu \mathrm{m}$, and the control and parasitic electrodes are $57.7 \mu \mathrm{m}$ by $115.5 \mu \mathrm{m}$ and $135 \mu \mathrm{m}$ by $270 \mu \mathrm{m}$ respectively, corresponding to $\rho=3$. 


\section{CHAPTER 4 CONCLUSIONS}

This thesis is a continuation of research reported in [6], [7],[8] and [9]. Following those works, it investigates two types of passivity based controllers. The system under study is a 1-DOF electrostatic piston mode actuator in the presence of parasitic capacitances due to an underlying conductive layer . The controllers based on the total charge can stabilize the structure with parasitics when the drive electrode area is large enough to screen the parasitic electrode from the movable electrode. However, when the drive electrode area is smaller than both the movable and parasitic electrode areas, the controller based on total charge may lose stability through subcritical Hopf bifurcation. furthering addition, the practical implementation of the controller becomes doubtful. Redesign of a passivity-based controller often involves selection of a different output. Therefore, a controller based on a feasible measurement, called here $\tilde{Q}_{c c}$, was formulated in order to resolve the issues on the controllers based on total charge. It is proved that the new controller based on $\tilde{Q}_{c c}$ can globally asymptotically stabilize the structure for the case where the drive electrode area is large enough to screen the parasitic electrode from the movable electrode. For the case where the drive electrode area is smaller than both the movable and parasitic electrode areas, it is shown by numerical simulations that the controller based on $\tilde{Q}_{c c}$ has a large (perhaps global) region of attraction for all configurations of practical interest. Finite-element simulations on $\tilde{Q}_{c c}$ control show that the parallel plate approximation used in controller design may introduce significant offsets in the steady state position of the movable electrode, with most of this due to the dynamic inversion component. This effect can be reduced by using more accurate capacitance models. 
Texas Tech University, Imiya Manjula Wickramasinghe, May 2008

\section{BIBLIOGRAPHY}

[1] R. C. Anderson, B. Kawade, D. H. S. Maithripala, K. Ragulan, J. M. Berg, R. O. Gale, "Integrated charge sensors for feedback control of electrostatic MEMS," Proceedings of the SPIE conference on Smart Structures and Materials 2005: Sensors and Smart Structures Technologies for Civil, Mechanical, and Aerospace Systems, San Diego, 6-10 March 2005.

[2] ANSYS Users Guide, Release 10.0A1, ANSYS, Inc., 2006

[3] C. I. Byrnes, A. Isidori and J. C. Willems, "Passivity, Feedback Equivalence, and the Global Stabilization of Minimum Phase Nonlinear Systems," IEEE Transactions on Automatic Control, (36) No. 11, pp. 1228 - 1240, Nov 1991.

[4] E. K. Chan, R. W. Dutton, "Electrostatic micromechanical actuator with extended range of travel,", Journal of Microelectromechanical Systems, Vol. 9, No. 3, pp. 321-328, 2000.

[5] D. Elata, O. Bochobza-Degni and Y. Nemirovsky, "Analytical Approach and Numerical Alpha-Line Method for Pull-In Hyper-Surface Extraction of Electrostatic Actuators with Multiple Uncoupled Voltage Sources," Journal of Microelectromechanical Systems, Vol. 12, No. 5, pp. 681-691, Oct 2003.

[6] D. H. S. Maithripala, J. M. Berg and W. P. Dayawansa, "Control of an Electrostatic MEMS Using Static and Dynamic Output Feedback," ASME Journal of Dynamical Systems Measurement and Control, Vol. 127, pp. 443 $450,2005$.

[7] D. H. S. Maithripala, B. D. Kawade, J. M. Berg, and W. P. Dayawansa, "A General Modeling and Control Framework for Electrostatically-Actuated Mechanical Systems," International Journal of Robust and Nonlinear Control, Special Issue on Control at the Nanoscale, Vol. 15, pp. 839 - 857, published online 15 September 2005. 
[8] Jordan M. Berg, D. H. S. Maithripala, B. D. Kawade, and W. P. Dayawansa, "Integrated Modeling and Control of Electrostatic MEMS, Part I: Modeling," Proceedings of the IEEE International Conference on Information and Automation, December 15-18, 2005, Colombo, Sri Lanka.

[9] D. H. S. Maithripala, B. D. Kawade, J. M. Berg and W. P. Dayawansa, "Integrated Modeling and Control of Electrostatic MEMS, Part II: Control," Proceedings of the IEEE International Conference on Information and Automation, December 15-18, 2005, Colombo, Sri Lanka.

[10] D. H. S. Maithripala, B. D. Kawade, I. P. M. Wickramasinghe, J. M. Berg, and W. P. Dayawansa, "Equilibrium Structure of a 1-DOF Electrostatic MEMS Model with Parasitics," Proceedings of the 2007 ASME International Mechanical Engineering Congress and Exposition, November 11-15, 2007, Seattle, USA.

[11] R. Nadal-Guardia, A. Dehe, R. Aigner and L. MĊastaner, "Current drive methods to extend the range of travel of electrostatic microactuators beyond the voltage pull-in point," Journal of Microelectromechanical Systems, Vol. 11, No. 3, pp. 255-263, June 2002.

[12] M. Napoli, W. Zhang, K. Turner and B. Bamieh, "Characterization of Electrostatically Coupled Microcantilevers," Journal of Microelectromechanical Systems, Vol. 14, No. 2, pp. 295-304, April 2005.

[13] Y. Nemirovsky and O. Bochobza-Degni, "A Methodology for the Pull-In Parameters of Electrostatic Actuators," Journal of Microelectromechanical Systems, Vol. 10, No. 4, pp. 601-615, Dec 2001.

[14] R. Ortega, A. J. van der Schaft, I. Mareels, and B. Maschke, "Putting Energy Back in Control," IEEE Control Systems Magazine, April, pp. 18-33, 2001.

[15] J. I. Seeger and B. E. Boser, "Charge Control of Parallel-Plate, Electrostatic Actuators and the Tip-In Instability," Journal of Microelectromechanical Systems, Vol. 12, No. 5, pp. 656-671, October 2003.

[16] Stephen D. Senturia, Microsystem Design, Kluwer Academic Publishers, Norwell, MA, 2001.

[17] G. C. Zhu, J. Levine, L. Praly, and Y. A. Peter, "Flatness-based control of electrostatically actuated MEMS with application to adaptive optics: A simulation study," Journal of Microelectromechanical Systems, Vol. 15, No. 5, pp. 1165-1174, 2006. 
[18] G. C. Zhu, J. Penet, and L. Saydy, "Modeling and Control of Electrostatically Actuated MEMS in the Presence of Parasitics and Parametric Uncertainties," Journal of Dynamic Systems, Measurement, and Control, in press.

[19] B. D. Kawade, D. H. S. Maithripala, and J. M. Berg, "Efficient Multi-Physics Transient Analysis Incorporating Feedback-Dependent Boundary Conditions," Proceedings of the 2006 ANSYS Users Conference.

[20] G. Zhu, J. Levine, and L. Praly, "On the Differential Flatness and Control of Electrostatically Actuated MEMS," Proceedings of the $A C C$, Portland, Oregon, 2005.

[21] G. Flores, G. Mercado, J. A. Pelesco and N. Smyth, "Analysis of the Dynamics and Touchdown in a Model of Electrostatic MEMS," SIAM Journal of Applied Mathematics, Vol. 67, No. 2, pp. 434-446, Sep 2006.

[22] Steven T. Pattona and Jeffrey S. Zabinski, "Effects of dielectric charging on fundamental forces and reliability in capacitive microelectromechanical systems radio frequency switch contacts," Journal of Applied Physics,published online May 2006.

[23] Gregory N. Nielson and George Barbastathis, "Dynamic Pull-In of Parallel-Plate and Torsional Electrostatic MEMS Actuators," Journal of Microelectromechanical Systems, Vol. 15, No. 4 pp. 811 - 821, Aug 2006.

[24] I. P. M. Wickramasinghe, D. H. S. Maithripala, B. D. Kawade, J. M. Berg, and W. P. Dayawansa, "Passivity-Based Stabilization of a 1-DOF Electrostatic MEMS Model with a Parasitic Capacitance," IEEE Transactions on Control Systems Technology, in press. 


\section{APPENDIX A}

The formulations for Tabel I is as shown below.

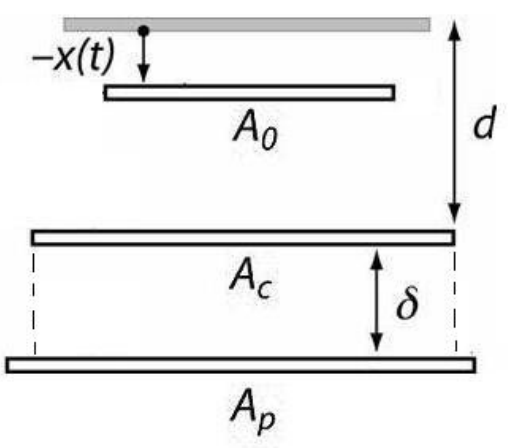

Case I

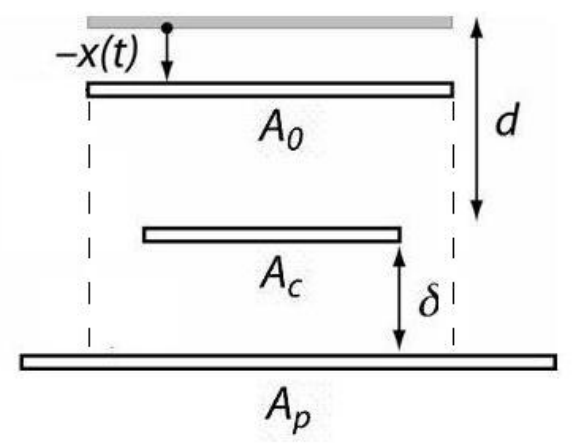

Case II

Figure 4.1. Configurations for Case I and Case II

For Case I Charge and voltage are related by

$$
\left[\begin{array}{l}
Q_{c} \\
Q_{p}
\end{array}\right]=\left[\begin{array}{cc}
\frac{\epsilon A_{0}}{(x+d)}+\frac{\epsilon A_{c}}{\delta} & -\frac{\epsilon A_{c}}{\delta} \\
-\frac{\epsilon A_{c}}{\delta} & \frac{\epsilon A_{c}}{\delta}
\end{array}\right]\left[\begin{array}{c}
V_{c} \\
V_{p}
\end{array}\right]
$$

Equation (4.1) is based on the infinite parallel plate capacitor model. Taking the inverse of the matrix of capacitance, one can obtain the relationship below

$$
\left[\begin{array}{c}
V_{c} \\
V_{p}
\end{array}\right]=\frac{(x+d)}{\epsilon A_{0}}\left[\begin{array}{cc}
1 & 1 \\
1 & 1+\frac{\rho \delta}{(x+d)}
\end{array}\right]\left[\begin{array}{l}
Q_{c} \\
Q_{p}
\end{array}\right]
$$

Simillarly for Case II

$$
\left[\begin{array}{l}
Q_{c} \\
Q_{p}
\end{array}\right]=\left[\begin{array}{cc}
\frac{\epsilon A_{c}}{(x+d)}+\frac{\epsilon A_{c}}{\delta} & -\frac{\epsilon A_{c}}{\delta} \\
-\frac{\epsilon A_{c}}{\delta} & \frac{\epsilon\left(A_{0}-A_{c}\right)}{(x+d+\delta)}+\frac{\epsilon A_{c}}{\delta}
\end{array}\right]\left[\begin{array}{c}
V_{c} \\
V_{p}
\end{array}\right]
$$


$\left[\begin{array}{l}V_{c} \\ V_{p}\end{array}\right]=\frac{(x+d)}{\epsilon A_{0}}\left[\begin{array}{cc}1+\frac{\delta(\rho-1)}{x+d+\delta} & 1 \\ 1 & 1+\frac{\delta}{x+d}\end{array}\right]\left[\begin{array}{l}Q_{c} \\ Q_{p}\end{array}\right]$ 


\section{APPENDIX B}

\section{The Main Program}

This is the Matlab code for the main MATLAB program that we used to generate the simulation shown in the Chapter 3.

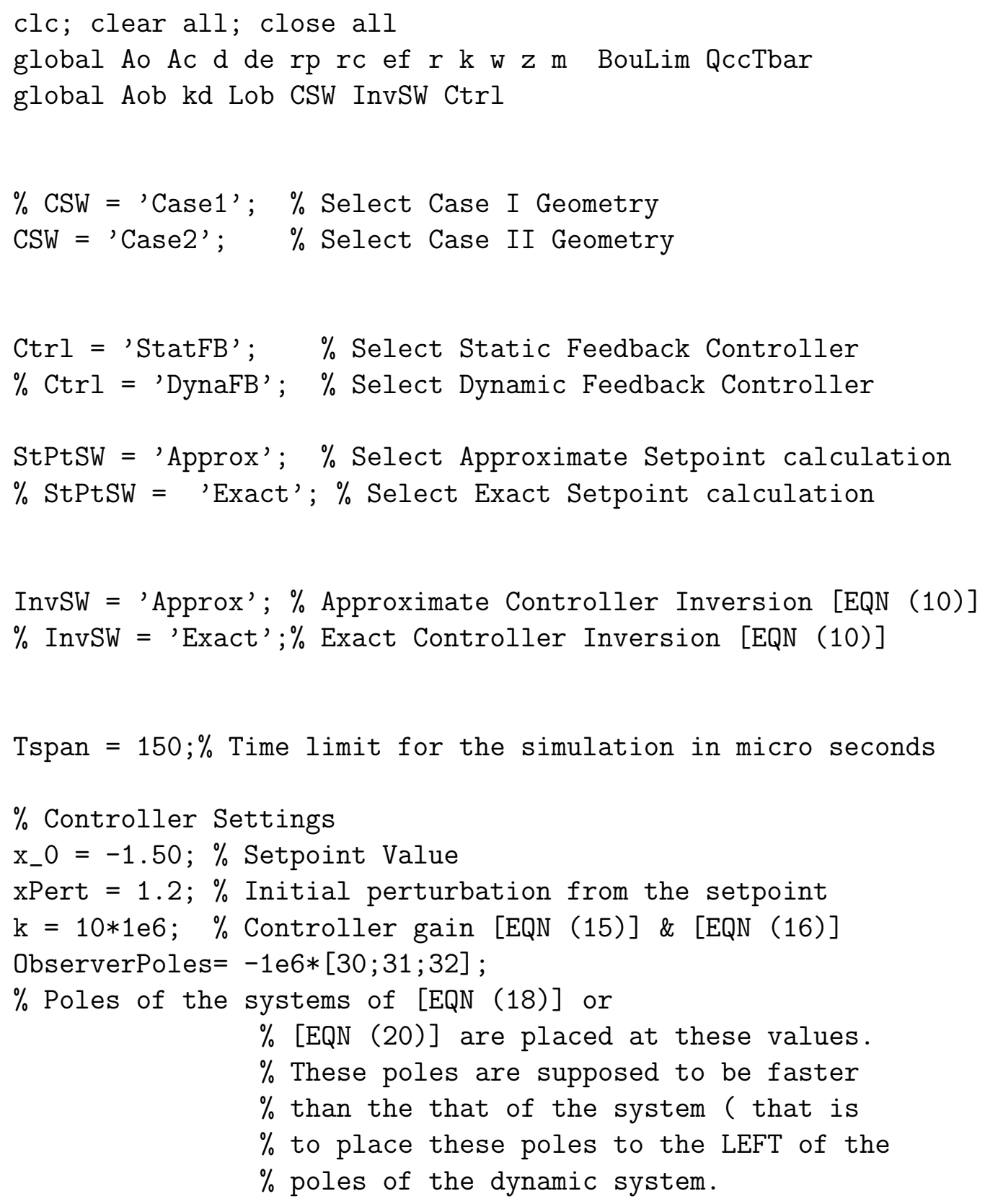


$\%$ Geometric Parameters

$\mathrm{d}=3 ; \%$ Zero voltage gap $\mathrm{b} / \mathrm{w}$ movalbe ele: and drive ele:

de $=.5 ; \%$ Gap $b / \mathrm{w}$ drive ele: and parasitic ele:

$r=3 ; \%$ This value may be used in the theoretical

$\%$ model calculations

Ao $=200 * 100 ; \%$ Moving electrode area in square micrometers

$\%$ Mechanical Parameters

$\mathrm{m}=$ Ao*. $8 * 2700 \mathrm{e}-18 ; \%$ Mass of movable electrode in kilograms

$z=1 e-2 * 8.6 ; \%$ Value of damping ratio 'ZETA' in [EQN (5)]

$\mathrm{w}=\operatorname{sqrt}(7.05 / \mathrm{m}) ; \%$ Value of angular frequency

$$
\% \text { 'OMEGA' in [EQN (5)] }
$$

$\%$ Electrical Parameters

ef $=8.85418782 \mathrm{e}-6$; \% Permitivity of teh medium 'EPSILON'

$r c=1 e-6 ; \%$ Value of rcc in [EQN (1)]

$r p=1 e-6 ; \%$ Value of $r p p$ in [EQN (3)]

$\%$ Simulation Control

$\%$ This specifies the controls of ODE45 solver

OPTIONS =odeset ('Events', @Events, 'AbsTol' , 1e-6, ...

'RelTol', 1e-6, 'MaxStep' ,10);

Ac $=A o / r ; \%$ Control electrode area

BouLim $=-d+d * .011$;

$\%$ Value of $\mathrm{x}$ at which movable electrode hit control

$\%$ electrode. Small margin is specified for dielectric

$\%$ coating

$\%$ Initializations

Xob0 $=[] ; \%$ Vector of Observer States

$\mathrm{T}=[] ; \%$ Vector of simulation time values

$\mathrm{Y}=[] ; \%$ Vector of simulation output values

Tstart $=0 ; \%$ Simulation start time

$\mathrm{Tc}=\mathrm{Tstart} ; \%$ Intermediate simulation start time variable

if strcmp(Ctrl, 'DynaFB') \% Invokes only in Dynamic Feedback Control

$\%$ Observer $\mathrm{Xdot}=\mathrm{Aob} * \mathrm{X}+\mathrm{F}(\mathrm{Y})+\mathrm{L}(\mathrm{Xme}-\mathrm{Xob})$

$\mathrm{Aob}=\left[0,0,0 ; 0,0,1 ; 0,-\mathrm{w}^{\wedge} 2,-2 * \mathrm{z}_{\mathrm{w}}\right] ; \%$ Aob Matrix 


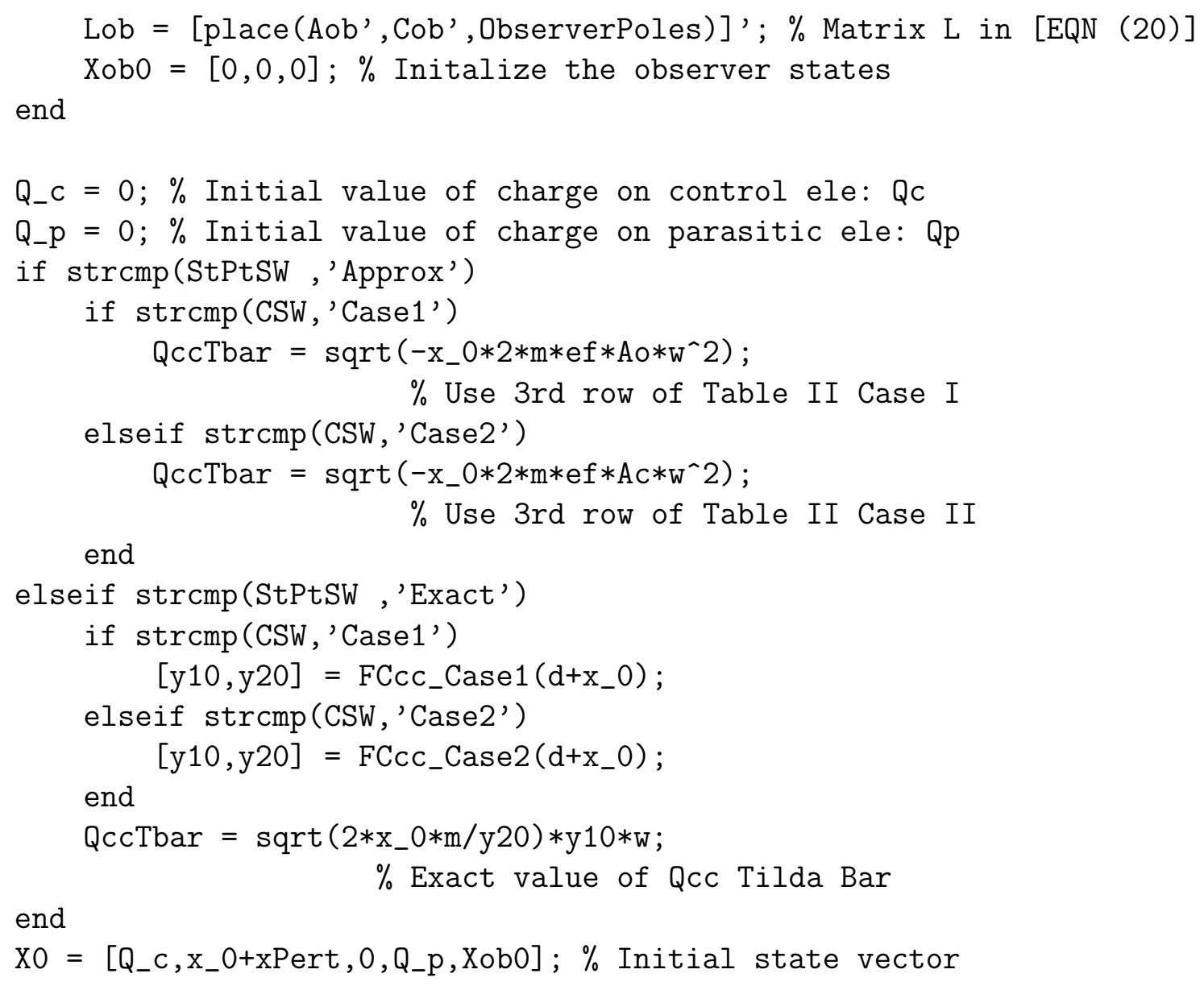




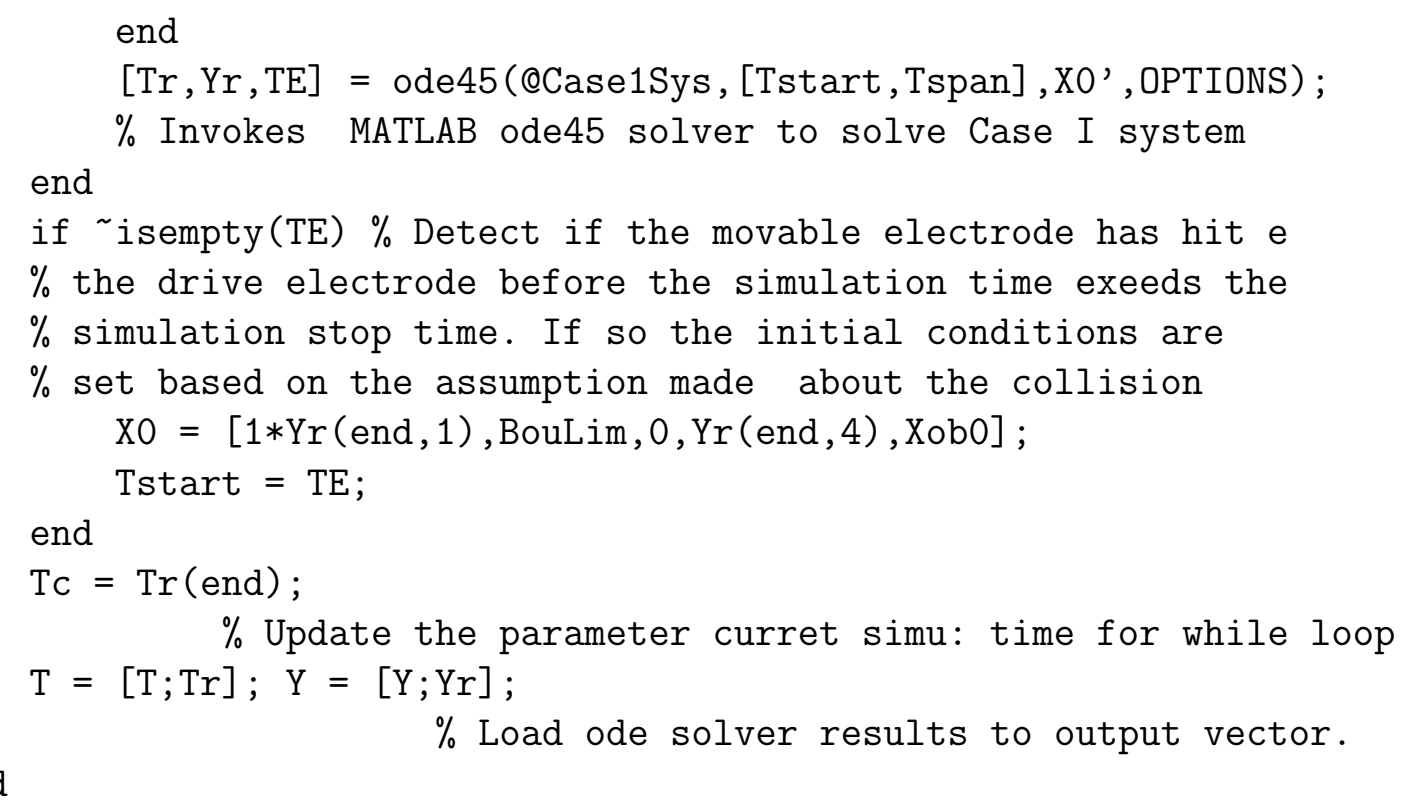

figure \% Open a figure

hold on $\%$ Set figure properties to add lines plot (T, $Y(:, 2)+d,{ }^{\prime}$ ', 'LineWidth',3) \% Plot electrode gap variation plot $\left(\mathrm{T}\right.$, ones $\left.(\operatorname{size}(\mathrm{Y}(:, 2))) *\left(\mathrm{x}_{-} 0\right)+\mathrm{d},{ }^{\prime}-\mathrm{k}^{\prime}\right) \%$ Plot setpoint line plot $\left(\mathrm{T}\right.$, ones $(\operatorname{size}(\mathrm{Y}(:, 2))) *(0)+\mathrm{d},{ }^{\prime}-. \mathrm{k}$ ' , 'LineWidth', 2)

$\%$ Plot Zero volt position

$\operatorname{plot}\left(\mathrm{T}\right.$, ones $(\operatorname{size}(\mathrm{Y}(:, 2))) *(0),{ }^{\prime}: \mathrm{k}^{\prime}$, , LineWidth', 2$)$

$\%$ Drive electrode position

plot (T, ones $(\operatorname{size}(Y(:, 2))) *$ BouLim+d, ' $: k^{\prime}$, ' LineWidth', .5)

$\%$ Plot collision limit

hold off $\%$ Set figure properties back to overwrite mode legend('Electrode Gap ', 'Setpoint', 'Zero Voltage Position'...

\%Add Legend ,'Drive Electrode');

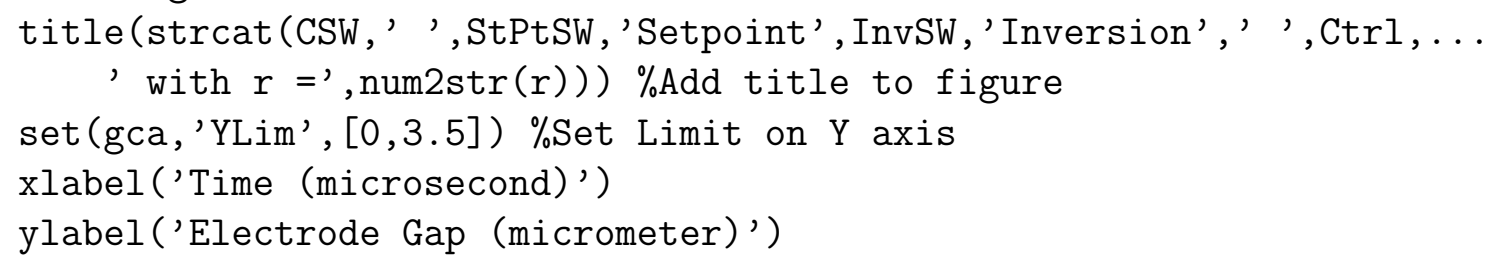




\section{APPENDIX C}

\section{The Sub Programs}

These subprograms must be saved with the repestive file names in the current directory, where the main program is saved.

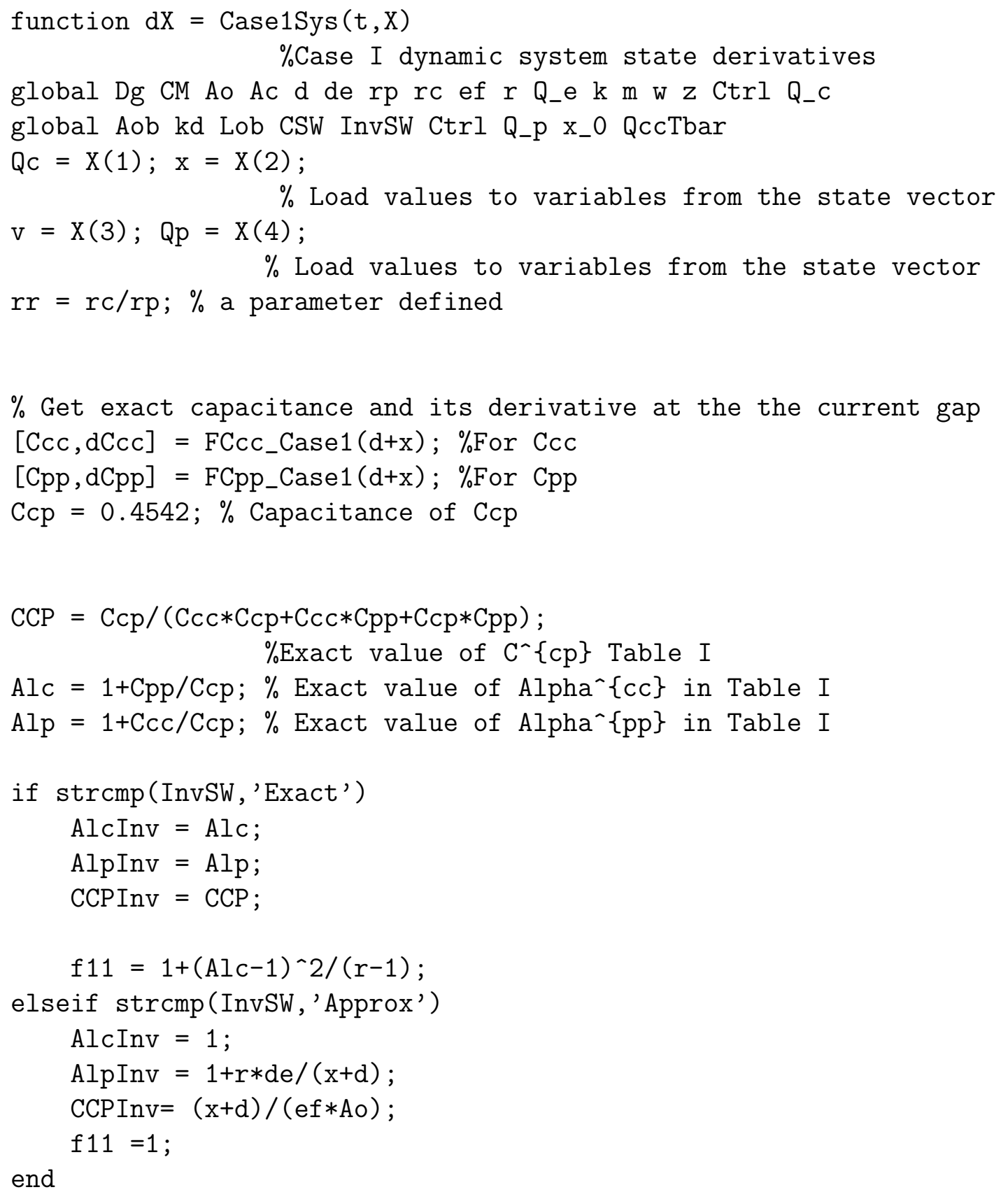




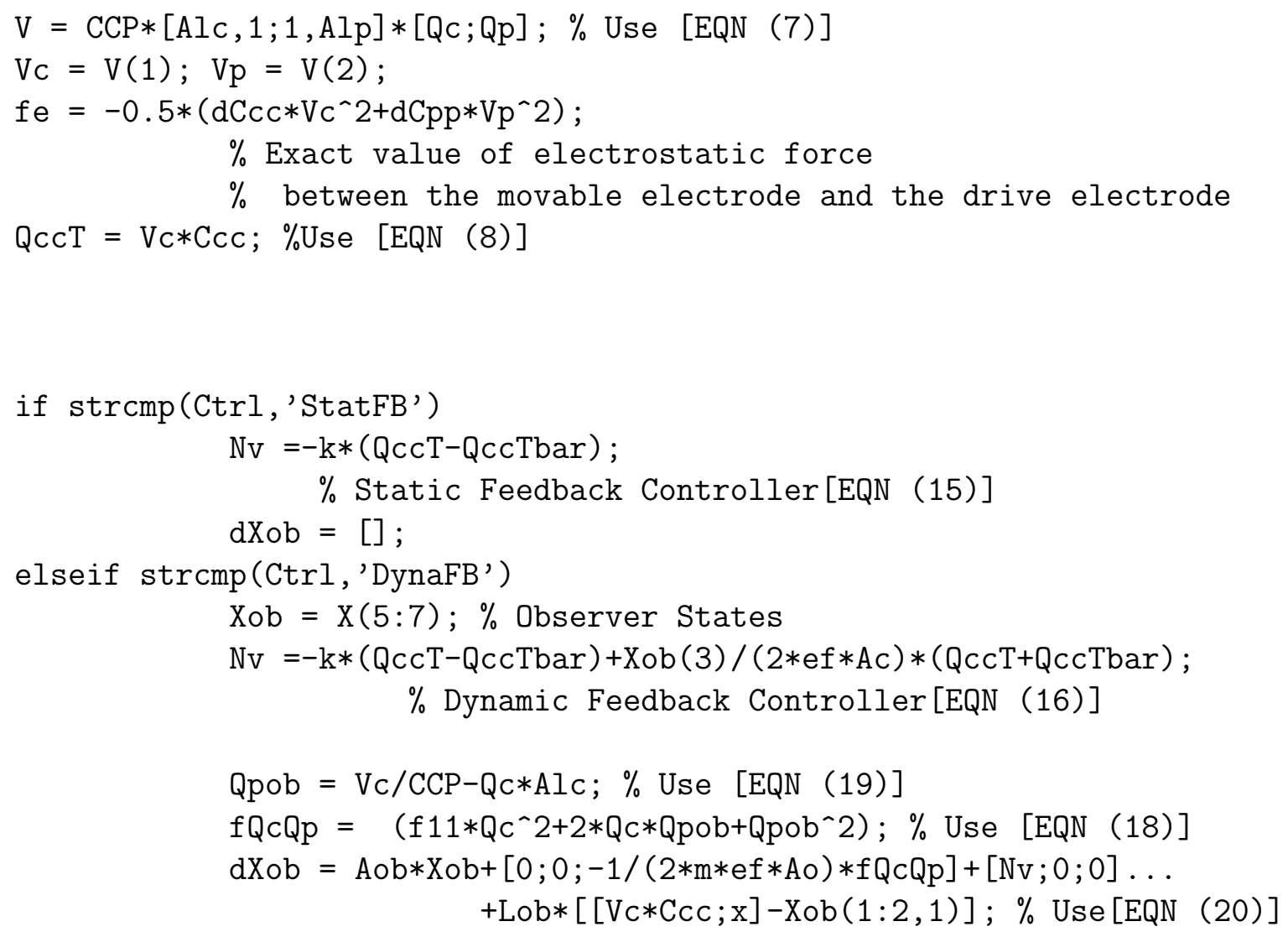




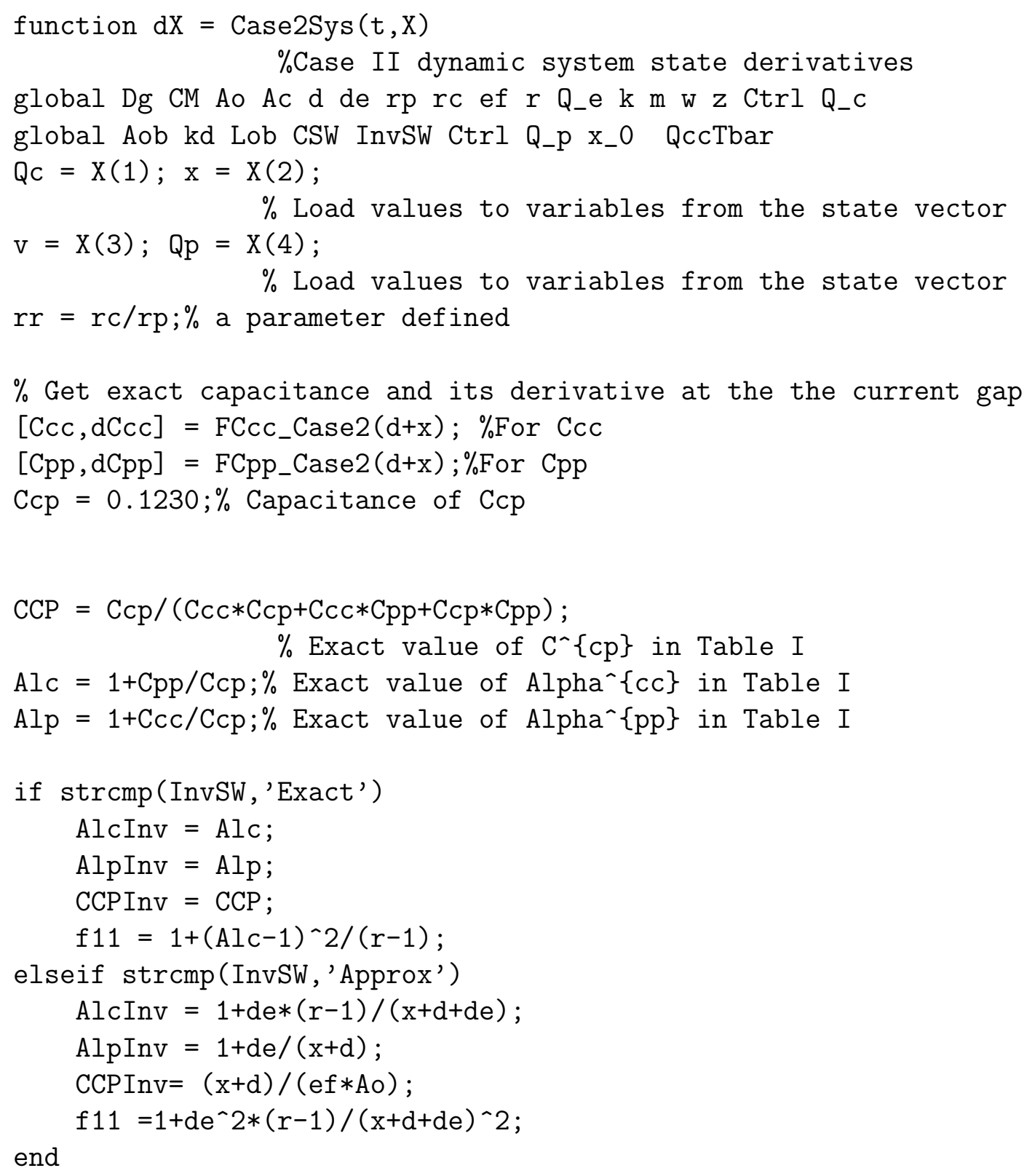




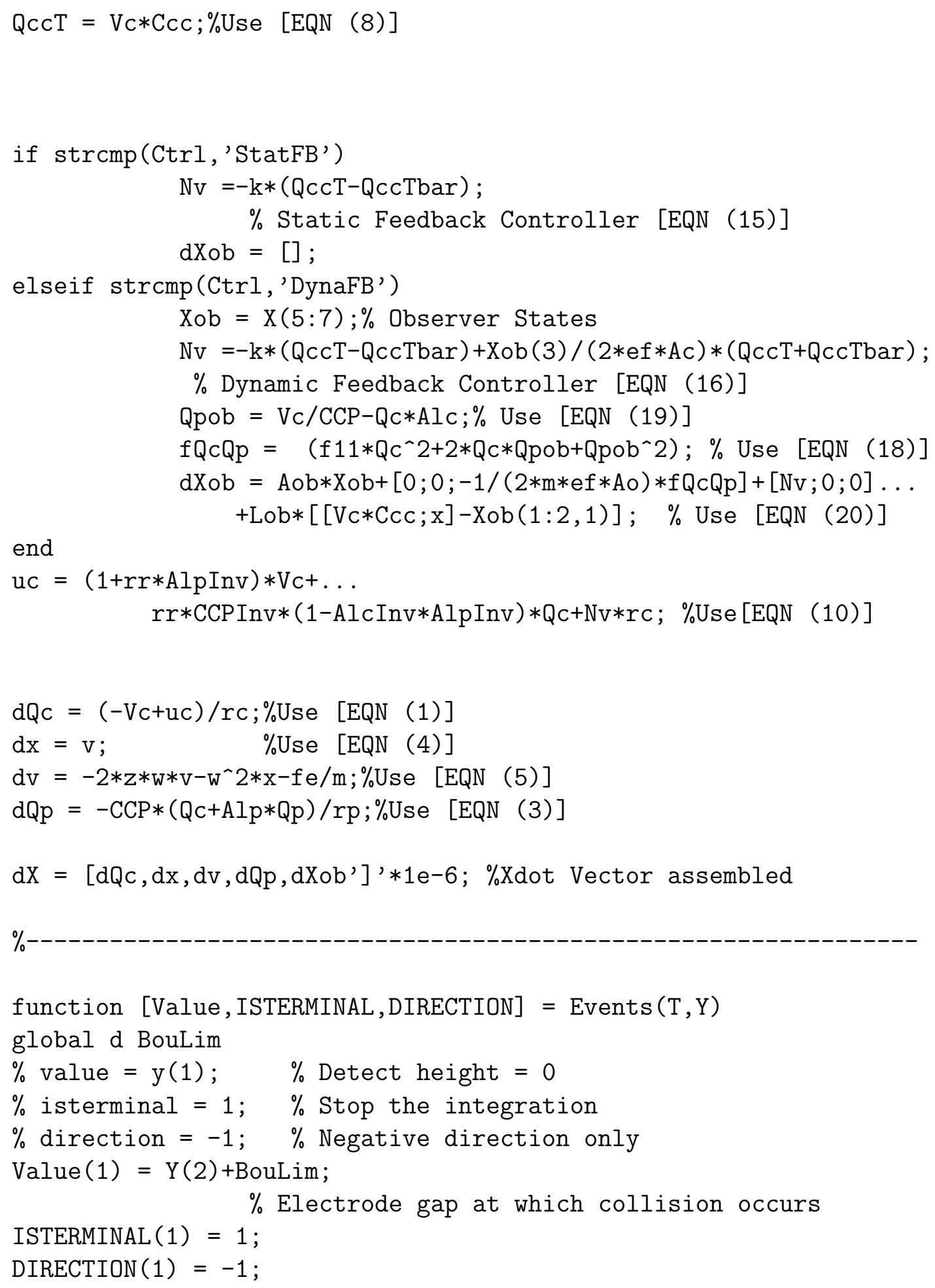




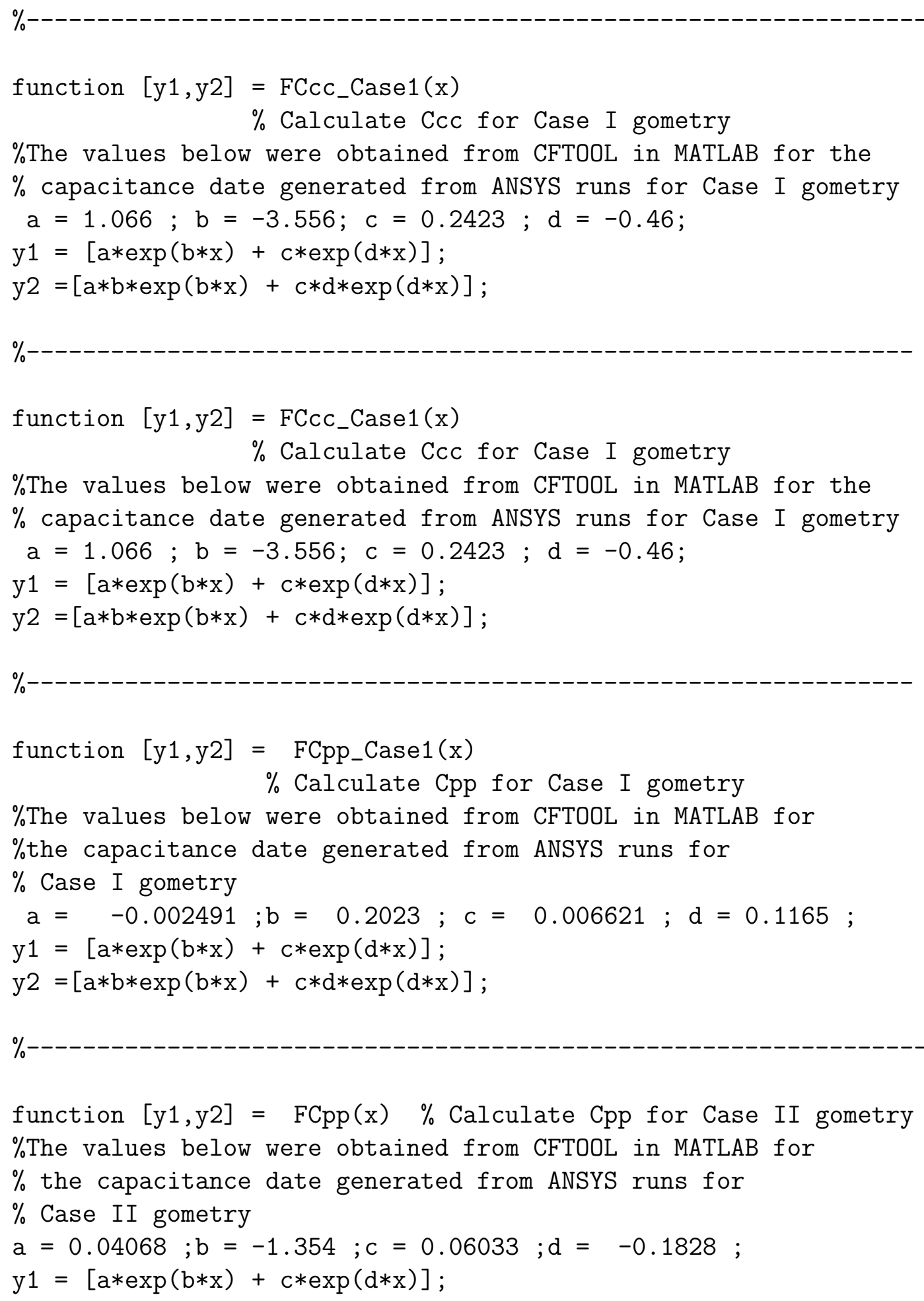


y2 $=[a * b * \exp (b * x)+c * d * \exp (d * x)] ;$ 


\section{PERMISSION TO COPY}

In presenting this thesis in partial fulfillment of the requirements for a master's degree at Texas Tech University or Texas Tech University Health Sciences Center, I agree that the Library and my major department shall make it freely available for research purposes. Permission to copy this thesis for scholarly purposes may be granted by the Director of the Library or my major professor. It is understood that any copying or publication of this thesis for financial gain shall not be allowed without my further written permission and that any user may be liable for copyright infringement.

Agree (Permission is granted.)

Imiya Manjula Wickramasinghe

Student Signature

Disagree (Permission is not granted.)
April 23, 2008

Date 